\title{
EXAMINING THE SEYFERT-STARBURST CONNECTION WITH ARCSECOND-RESOLUTION RADIO CONTINUUM OBSERVATIONS
}

\author{
SePpo Laine \\ Spitzer Science Center, California Institute of Technology, Mail Code 220-6, \\ Pasadena, CA 91125; seppo@ipac.caltech.edu \\ JARI K. Kotilainen \\ Tuorla Observatory, University of Turku, Väisäläntie 20, 21500 Piikkiö, Finland \\ Juha Reunanen \\ Department of Astronomy, University of Leiden, P.O. Box 9513, 2300 RA Leiden, Netherlands \\ StUART D. RYDER \\ Anglo-Australian Observatory, P.O. Box 296, Epping, NSW 1710, Australia \\ AND \\ RAINER BECK \\ Max-Planck-Institut für Radioastronomie, Auf dem Hügel 69, 53121 Bonn, Germany \\ Received 2004 November 1; accepted 2005 October 14
}

\begin{abstract}
We compare the arcsecond-scale circumnuclear radio continuum properties of five Seyfert and five starburst galaxies, concentrating on the search for any structures that could imply a spatial or causal connection between the nuclear activity and a circumnuclear starburst ring. No evidence is found in the radio emission for a link between the triggering or feeding of nuclear activity and the properties of circumnuclear star formation. Conversely, there is no clear evidence of nuclear outflows or jets triggering activity in the circumnuclear rings of star formation. Interestingly, the difference in the angle between the apparent orientation of the most elongated radio emission and the orientation of the major axis of the galaxy is on average larger in Seyfert galaxies than in starburst galaxies, and Seyfert galaxies appear to have a larger physical size scale of the circumnuclear radio continuum emission. The concentration, asymmetry, and clumpiness parameters of radio continuum emission in Seyfert galaxies and starbursts are comparable, as are the radial profiles of radio continuum and near-infrared line emission. The circumnuclear star formation and supernova rates do not depend on the level of nuclear activity. The radio emission usually traces the near-infrared $\mathrm{Br} \gamma$ and $\mathrm{H}_{2} 1-0 S(1)$ line emission on large spatial scales, but locally their distributions are different, most likely because of the effects of varying local magnetic fields and dust absorption and scattering.
\end{abstract}

Key words: galaxies: active — galaxies: nuclei — galaxies: Seyfert — galaxies: starburst — radio continuum: galaxies

\section{INTRODUCTION}

Up to $10 \%$ of nearby galaxies have energetic, nonstellar Seyfert activity in their nuclei (e.g., Ho et al. 1997a). Currently, it is unknown why this fraction is $10 \%$ and not $100 \%$. One of the most appealing explanations for this puzzle is the duty cycle hypothesis (e.g., Eracleous et al. 1995). It can be speculated that all spiral galaxies (Seyfert activity appears almost exclusively in spiral galaxies) become active for a limited period of time in their lives. Since a typical age for a nearby spiral galaxy is $10^{10} \mathrm{yr}$ and $10 \%$ of nearby spirals have Seyfert nuclei, the activity may last up to $10^{9} \mathrm{yr}$.

The central problem in feeding active galactic nucleus (AGN) activity is the supply of adequate fuel. While the tidal disruption of stars in the vicinity of supermassive nuclear black holes is unlikely to be efficient enough to supply several solar masses per year, as is required for feeding quasar activity, the mass consumption rates in Seyfert galaxies are only on the order of $0.1 M_{\odot} \mathrm{yr}^{-1}$ (e.g., Bian \& Zhao 2003). The tidal disruption of stars may be a sufficient mechanism for maintaining LINER activity (Eracleous et al. 1995), but the only viable mechanism for feeding Seyfert activity appears to be the conversion of gas mass into radiation in the accretion disks (Shlosman et al. 1990) that have been speculated to surround supermassive nuclear black holes. In addition to the possibility of nuclear bars, or "bars within bars," magnetic braking in a circumnuclear ring can drive gas inflow toward a nucleus and its accretion disk (Beck et al. 1999, 2005).

Interstellar gas in the central region of a galaxy can come from at least two sources. First of these is mass loss from evolved stars. This mechanism may produce a few times $0.1 M_{\odot} \mathrm{yr}^{-1}$ within the central few kiloparsecs (e.g., Faber \& Gallagher 1976). Another source of gas is from secular inflow, especially in a barred potential. Gravitational torques from bars are known to be capable of extracting angular momentum from the gas that piles up along the leading edge of the bar and causing a secular inflow of gas toward the central region (e.g., Simkin et al. 1980; Noguchi 1988; Barnes \& Hernquist 1991). If a dynamical resonance (the so-called inner Lindblad resonance [ILR]; e.g., Contopoulos 1975; Athanassoula 1992) exists, gas will tend to pile up in a ring around the ILR radius (there can be zero, one, or two ILRs or, in the case of a large central mass concentration, even a third ILR; gas tends to pile up and form a ring between the two outer ILRs, e.g., Knapen et al. 1995). Properties of such rings have been discussed in numerous papers. A good and comprehensive summary is given by Buta \& Combes (1996).

The nuclear rings are often locations of vigorous star formation (SF). While the exact fraction of gas that is consumed by SF 
in these rings is not known yet (although it is likely to vary from galaxy to galaxy), it is clear that the circumnuclear rings play a crucial role in regulating the gas supply to the nucleus (e.g., Heller \& Shlosman 1996; Regan et al. 1999). Therefore, it is of great importance to determine whether some properties of the circumnuclear rings, such as a special location of star-forming regions, SF rates, closeness to the nucleus, etc., are related to the activity class of the nucleus. Conversely, nuclear activity can have an impact on the properties of the starburst ring, e.g., in the form of star-forming activity in the circumnuclear ring triggered by a radio jet, as demonstrated on a large scale in Minkowski's Object (e.g., Fragile et al. 2004). These issues can be studied by looking for similarities and/or differences in the ring properties and the strength of nuclear activity between Seyfert and starburst galaxies. Earlier studies of circumnuclear rings have largely concentrated on the use of ultraviolet, optical, or near-infrared (NIR) images and spectra to study the properties of SF in these regions (e.g., Benedict et al. 1993; Barth et al. 1995; Kotilainen et al. 1996a, 1996b, 2000, 2001; Elmegreen et al. 1997, 1998, 1999; Buta et al. 1999, 2000; Pérez-Ramírez et al. 2000; Reunanen et al. 2000; Maoz et al. 2001; Alonso-Herrero et al. 2001; Ryder et al. 2001). Direct observations of the molecular gas also exist (e.g., Sakamoto et al. 1995; Benedict et al. 1996), mostly via CO that can be used as a tracer of the more abundant molecular hydrogen with certain caveats, such as the uncertainty in the conversion of the CO luminosities into total molecular gas masses, but the spatial resolution in most $\mathrm{CO}$ studies has been insufficient to resolve the rings adequately.

An alternative and potentially more powerful way to study the properties of the circumnuclear rings is provided by highresolution radio continuum observations. Radio wavelengths are free of extinction effects that dust has in optical and, for high extinction, even in NIR images. The radio emission coming from the rings is believed to consist of two main components: first, thermal emission that traces bremsstrahlung from free electrons in $\mathrm{H}$ II regions and is thermalized by the optically thick medium, and second, nonthermal emission that comes from electrons spiraling in magnetic fields, either surrounding recent supernova (SN) remnants (seen in high-resolution maps), in the general magnetic field of the underlying disk galaxy (seen in low-resolution, high-sensitivity maps; e.g., Turner \& Ho 1994), or in the enhanced magnetic field of the ring (Beck et al. 2005). Emission from AGNs is also nonthermal. Nonthermal emission usually dominates the emission maps seen at 6 and $20 \mathrm{~cm}$, whereas at shorter wavelengths the thermal fraction can be substantial or even dominate (e.g., Turner \& Ho 1994).

An interesting feature of radio continuum maps of several active galaxies is the strong point source in the nucleus and jets or highly collimated outflows emanating from the nucleus. In Seyfert galaxies, these jets are usually seen at a scale of a few hundred parsecs (Schmitt et al. 2001), but they can extend to kiloparsec scales (e.g., NGC 4258: van Albada \& van der Hulst 1982; NGC 7479: Laine \& Gottesman 1998). The orientation of the jets with respect to the rotation axis or disk plane of the galaxy has been observed to be fairly random (Schmitt et al. 1997). Such a collimated outflow has the potential to trigger SF in a circumnuclear region, as hinted at by, e.g., Bransford et al. (1998) and as seen in Minkowski's Object (Fragile et al. 2004). The recent study by Knapen (2005) suggests that galaxies with an active nucleus may have rings more often than galaxies without such a nucleus. One of the main objectives of the current paper is to investigate whether evidence for jets and the triggering of SF exists among nearby Seyfert galaxies and to contrast their circumnuclear properties with those of nearby starburst galaxies.
The triggering of SF in starburst galaxies is most likely external to the circumnuclear region, as there is no strong nuclear outflow component.

Several previous investigations of the radio continuum morphology in the central regions of nearby disk galaxies have been performed (e.g., Collison et al. 1994; Saikia et al. 1994; Turner \& Ho 1994; Bransford et al. 1998; Forbes \& Norris 1998; Morganti et al. 1999; Beck et al. 2000, 2005; Nagar et al. 2000; Thean et al. 2000; Ho \& Ulvestad 2001). However, none of these earlier investigations had either a balanced sample between Seyfert and non-Seyfert galaxies or supporting NIR line images of $2.166 \mu \mathrm{m}$ $\mathrm{Br} \gamma$ or $2.122 \mu \mathrm{m} \mathrm{H}_{2} 1-0 S(1)$ emission with which to study the star-forming morphology and properties. Many of the previously listed studies also had resolution and sensitivity combinations that were not ideally suited to detecting extended but resolved circumnuclear structure, for which a resolution of order $1^{\prime \prime}-2^{\prime \prime}$ is needed, together with a sensitivity to structures tens of arcseconds in extent. Our new observations are ideal for the study of such structures. In addition, we have gathered data at several wavelengths, approximately matched in spatial resolution, to further improve the sensitivity to emission from different mechanisms at various spatial scales and to provide some information on the radio spectral indices at various locations in our targets.

\section{SAMPLE AND OBSERVATIONS}

Our sample consists of both Seyfert and starburst galaxies, all of which are either barred or merging systems, thus making recent gas inflow likely. We include all the nearby (within $100 \mathrm{Mpc}$ ) Seyfert, starburst, or merging/interacting galaxies that have NIR line images of $2.166 \mu \mathrm{m} \mathrm{Br} \gamma$ emission, $2.122 \mu \mathrm{m} \mathrm{H}_{2} 1-0 S(1)$ emission, or both available and that do not have existing sensitive radio continuum observations at $1^{\prime \prime}-2^{\prime \prime}$ resolution at $3.5,6$, or $20 \mathrm{~cm}$. Very Large Array (VLA) sensitivity in the $L$ band $(20 \mathrm{~cm})$ improved by a factor of 2 in the early 1990s when the receivers were upgraded (P. Lilly \& R. Perley 2005, private communication). The $C$-band receivers have also improved, resulting in a sensitivity improvement, although not as dramatic as in the $L$ band. Therefore, in some cases we reobserved galaxies that had no VLA radio continuum observations at a comparable resolution since the late 1980s.

The general parameters of the 10 galaxies that satisfied our sample selection criteria are listed in Table 1. Of our sample galaxies, five have Seyfert classifications (one Seyfert 1 and four Seyfert 2 galaxies), five have starburst nuclei, and at least two are in merging or interacting systems. A sample of this size is useful in a pilot study such as ours to look for any trends or differences between the various nuclear activity classes. We do not distinguish between the various activity subclasses such as Seyfert 1 and Seyfert 2 galaxies in the following but only contrast the properties of Seyfert galaxies against non-Seyfert galaxies.

VLA observations of the sample galaxies were taken during three separate observing sessions (see Table 2). The galaxies were mixed both among and within the two observing sessions to maximize the $u-v$ coverage. Weather conditions were excellent for the $6 \mathrm{~cm}$ run, while cloud cover was between $15 \%$ and $75 \%$ for the 3.5 and $20 \mathrm{~cm}$ runs. These wavelengths were selected because they give approximately comparable spatial resolutions when observed with the VLA in its A $(20 \mathrm{~cm}), \mathrm{B}(6 \mathrm{~cm})$, and $C(3.5 \mathrm{~cm})$ configurations. The $3.5 \mathrm{~cm}$ band was used instead of the $2 \mathrm{~cm}$ band because of its higher sensitivity. The large range of wavelengths also gives us some leverage to inspect differences in the distribution of thermal $(3.5 \mathrm{~cm})$ and nonthermal $(20 \mathrm{~cm})$ emission. The absolute flux calibration is based on 5-10 minute scans of 3C 48 and 3C 286. The target galaxy observations were 
TABLE 1

General Parameters of the Galaxy Sample

\begin{tabular}{|c|c|c|c|c|c|c|c|c|}
\hline $\begin{array}{l}\text { Galaxy } \\
\text { (1) }\end{array}$ & $\begin{array}{l}\text { Hubble Type } \\
\text { (2) }\end{array}$ & $\begin{array}{l}\text { Activity Type } \\
\text { (3) }\end{array}$ & $\begin{array}{c}V_{\text {hel }} \\
\left(\mathrm{km} \mathrm{s}^{-1}\right) \\
(4)\end{array}$ & $\begin{array}{c}D \\
(\mathrm{Mpc}) \\
(5)\end{array}$ & $\begin{array}{c}B_{T} \\
(\mathrm{mag}) \\
(6)\end{array}$ & $\begin{array}{l}\text { Bar Length } \\
\text { (kpc) } \\
\text { (7) }\end{array}$ & $\begin{array}{c}\text { Deprojected Bar } \\
\text { Ellipticity } \\
\text { (8) }\end{array}$ & $\begin{array}{c}\text { NIR Data Available } \\
\text { (9) }\end{array}$ \\
\hline 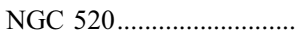 & S pec & Starburst (merger) & 2281 & 27.8 & 12.2 & $\ldots$ & $\ldots$ & $\mathrm{H}_{2}, \mathrm{Br} \gamma$ \\
\hline 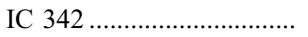 & $\mathrm{SAB}(\mathrm{rs}) \mathrm{cd}$ & Starburst & 31 & 3.9 & 9.1 & 1.7 & 0.43 & $\mathrm{H}_{2}, \mathrm{Br} \gamma, \mathrm{Fe}$ II \\
\hline 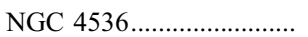 & $\mathrm{SAB}(\mathrm{rs}) \mathrm{bc}$ & Starburst & 1808 & 13.3 & 11.2 & 1.2 & 0.37 & $\mathrm{H}_{2}$ \\
\hline NGC $6240^{\mathrm{a}} \ldots \ldots \ldots \ldots \ldots \ldots$ & I0: pec & Sy2 (merger) & 7339 & 96.1 & 13.8 & $\ldots$ & $\ldots$ & $\mathrm{H}_{2}, \mathrm{Fe}$ II \\
\hline NGC $6574^{\mathrm{a}}$. & $\mathrm{SAB}(\mathrm{rs}) \mathrm{bc}$ & Sy2 & 2282 & 35.0 & 12.8 & 1.7 & 0.47 & $\mathrm{H}_{2}, \mathrm{Br} \gamma$ \\
\hline NGC $6764^{\mathrm{a}} \ldots \ldots \ldots \ldots \ldots \ldots \ldots$ & $\mathrm{SB}(\mathrm{s}) \mathrm{bc}$ & Sy2 & 2416 & 37.0 & 12.6 & 9.9 & 0.69 & $\mathrm{H}_{2}, \mathrm{Br} \gamma$ \\
\hline 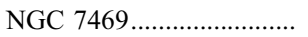 & $\left(\mathrm{R}^{\prime}\right) \mathrm{SAB}(\mathrm{rs}) \mathrm{a}$ & Sy1.2 & 4892 & 64.4 & 13.0 & 0.7 & 0.52 & $\mathrm{Br} \gamma, \mathrm{Fe}$ II \\
\hline 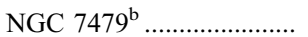 & $\mathrm{SB}(\mathrm{s}) \mathrm{c}$ & Sy2 & 2381 & 32.4 & 11.6 & 7.5 & 0.74 & $\mathrm{H}_{2}, \mathrm{Br} \gamma$ \\
\hline NGC $7714^{\mathrm{b}} \ldots \ldots \ldots \ldots \ldots \ldots \ldots \ldots$ & $\mathrm{SB}(\mathrm{s}) \mathrm{b}: \mathrm{pec}$ & Starburst & 2798 & 36.9 & 13.0 & 7.7 & 0.52 & $\mathrm{H}_{2}, \mathrm{Br} \gamma$ \\
\hline 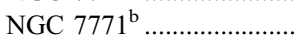 & $\mathrm{SB}(\mathrm{s}) \mathrm{a}$ & Starburst & 4277 & 56.4 & 13.1 & 8.8 & 0.62 & $\mathrm{H}_{2}, \mathrm{Br} \gamma$ \\
\hline
\end{tabular}

Notes._Col. (1): Galaxy names. Col. (2): Hubble type from NED. Col. (3): Activity or interaction class. Col. (4): Heliocentric velocity from NED. Col. (5): Distance

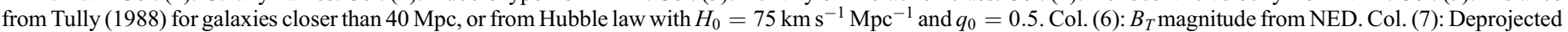

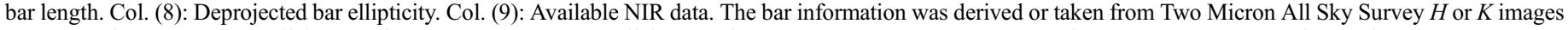

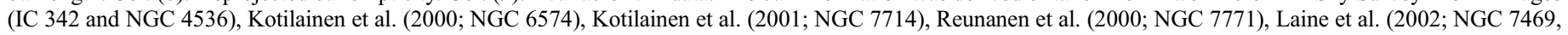
NGC 7479), and J. Kotilainen et al.(1998, unpublished; NGC 6764).

a Galaxy also observed at $3.5 \mathrm{~cm}$.

${ }^{b}$ Galaxy also observed at $6 \mathrm{~cm}$.

bracketed between 1 and 2 minute scans of nearby phase calibrators, and their fluxes were bootstrapped to those of the primary calibrators. The derived fluxes for the phase calibrators were then compared to values found in the VLA calibrator flux density database to check their reasonability. Uncertainties in the quoted fluxes are dominated by the uncertainty in setting the absolute flux scale and are estimated to be less than $10 \%$. Uncertainties in positions are estimated to be less than $0^{\prime \prime} .1$.

For maximum sensitivity we used both intermediate frequencies, with bandwidths of $50 \mathrm{MHz}$ and separated by $50 \mathrm{MHz}$. After minimal editing of the visibilities and calibration, we used the AIPS task imagr to Fourier transform the observed visibilities into brightness distribution maps on the sky. The "dirty" maps were deconvolved using the CLEAN procedure (Högbom 1974; Clark 1980) by placing "clean boxes" around the central area of the map (the galaxy) and around nearby background sources that may have had sidelobes that caused additional noise in the mapped field. The images were cleaned down to about 3 times the theoretical rms noise limit of the maps. We used no tapering and set the ROBUST parameter in imagr to zero, resulting in maps that are a compromise between maximum resolution and maximum sensitivity. Our typical synthesized beam sizes, tabulated in Table 3, have FWHM values around 1".3 at 6 and $20 \mathrm{~cm}$ and 2.5 at $3.5 \mathrm{~cm}$. We applied phase-only selfcalibration for the brightest sources in the sample, resulting in a slight improvement in the signal-to-noise ratio $(\mathrm{S} / \mathrm{N})$. The expected theoretical rms sensitivities were obtained in most cases in which sidelobe contamination from background sources is not severe.

\section{RESULTS}

\subsection{Radio Structure and Power}

Gray-scale and contour images of the radio continuum emission at 3.5, 6, and $20 \mathrm{~cm}$ from our sample galaxies are presented in Figure 1. The integration times and several other parameters of the final maps are given in Table 3 . Figure 2 shows the radial surface brightness profiles of $3.5,6$, and $20 \mathrm{~cm}$ radio continuum, $\mathrm{H}_{2} 1-0 \mathrm{~S}(1)$ emission, and $\mathrm{Br} \gamma$ emission. In Figures 3-8 we show the radio continuum emission together with either molecular hydrogen $\mathrm{H}_{2} 1-0 S(1)$ or $\mathrm{Br} \gamma$ emission maps, or both.

The morphology of the radio continuum emission varies from centrally peaked (NGC 520, NGC 7469, and NGC 7714) to elongated or ringlike emission (IC 342, NGC 4536, NGC 6240, NGC 6574, and NGC 7771) to outflows or jets (NGC 6764 and NGC 7479). The orientations of the circumnuclear radio continuum structure at $20 \mathrm{~cm}$ and the position angle of the main disk of the underlying galaxy are given in Table 4, together with the projected difference between the two and the inclination angle. The major axis of the radio continuum emission was determined by fitting a two-dimensional Gaussian to the brightest point after smoothing the image to $10^{\prime \prime}$ resolution and was used as the orientation of the radio emission. Most commonly the detected circumnuclear radio continuum structures are aligned close to the

TABLE 2

Parameters of the Vla Observing Runs

\begin{tabular}{|c|c|c|c|}
\hline Parameter & Session 1 & Session 2 & Session 3 \\
\hline 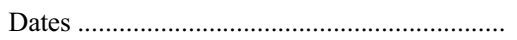 & 1999 Jul 25, 27 & 1999 Nov 26 & 2000 Jun $3-4$ \\
\hline 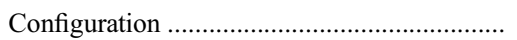 & $\mathrm{A}$ & B & $\mathrm{C}$ \\
\hline Maximum number of antennas........................... & 27 & 26 & 27 \\
\hline 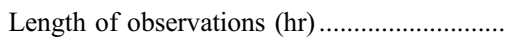 & $2.5,4$ & 3 & 2 \\
\hline Frequency $(\mathrm{GHz})$ & 1.4250 & 4.8601 & 8.4601 \\
\hline 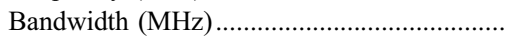 & 100 & 100 & 100 \\
\hline 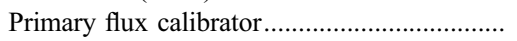 & $3 \mathrm{C} 286,3 \mathrm{C} 48$ & $3 \mathrm{C} 48$ & $3 \mathrm{C} 286$ \\
\hline Flux of primary flux calibrator (Jy) ................ & $14.554,16.319$ & 5.516 & 5.181 \\
\hline
\end{tabular}


TABLE 3

Observational and Measured Parameters

\begin{tabular}{|c|c|c|c|c|c|c|c|c|c|c|}
\hline \multirow[b]{3}{*}{ GaLAXY } & \multicolumn{5}{|c|}{ MAP PARAMETERS } & \multicolumn{5}{|c|}{ Source Parameters } \\
\hline & \multirow[b]{2}{*}{$\begin{array}{c}\lambda \\
(\mathrm{cm})\end{array}$} & \multirow[b]{2}{*}{$\begin{array}{l}\text { Integration Time } \\
\text { (s) }\end{array}$} & \multirow[b]{2}{*}{$\begin{array}{l}\text { Beam } \\
(\operatorname{arcsec})\end{array}$} & \multirow[b]{2}{*}{$\begin{array}{l}\text { P.A. } \\
\text { (deg) }\end{array}$} & \multirow[b]{2}{*}{$\begin{array}{c}\mathrm{rms} \\
\left(\mu \mathrm{Jy}_{\text {beam }}^{-1}\right)\end{array}$} & \multicolumn{2}{|c|}{ Total Flux (Jy) } & \multirow[b]{2}{*}{$\begin{array}{l}\text { Log Total Radio Power } \\
\left(\mathrm{W} \mathrm{Hz}^{-1}\right)\end{array}$} & \multirow[b]{2}{*}{$\begin{array}{l}\mathrm{U} / \mathrm{E}^{\mathrm{a}} \\
(\%)\end{array}$} & \multirow[b]{2}{*}{ Morphology } \\
\hline & & & & & & VLA & Single Dish & & & \\
\hline 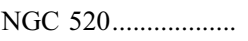 & 20 & 700 & $1.44 \times 1.33$ & 0 & 65 & 0.170 & 0.158 & 22.2 & 0 & $\mathrm{C}$ \\
\hline 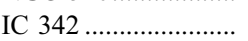 & 20 & 810 & $1.70 \times 1.07$ & 54 & 90 & 0.131 & 2.25 & 20.4 & 0 & $\mathrm{R}, \mathrm{E}$ \\
\hline NGC 4536.................. & 20 & 660 & $1.67 \times 1.42$ & 29 & 51 & 0.131 & 0.126 & 21.4 & 0 & $\mathrm{~L}, \mathrm{E}$ \\
\hline 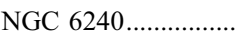 & 20 & 760 & $1.48 \times 1.33$ & 0 & 43 & 0.108 & 0.59 & 23.1 & 29 & $\mathrm{C}, \mathrm{R}$ \\
\hline NGC $6240 \ldots \ldots \ldots \ldots . . . .$. & 3.5 & 700 & $2.72 \times 2.28$ & 0 & 66 & 0.115 & $\ldots$ & 23.1 & 40 & $\mathrm{C}, \mathrm{R}$ \\
\hline 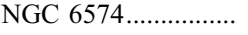 & 20 & 3590 & $1.34 \times 1.32$ & 0 & 42 & 0.030 & $\ldots$ & 21.6 & 4 & $\mathrm{C}, \mathrm{R}$ \\
\hline 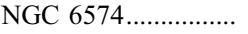 & 3.5 & 1940 & $2.33 \times 2.11$ & 0 & 29 & 0.026 & $\ldots$ & 21.6 & 2 & $\mathrm{C}, \mathrm{R}$ \\
\hline 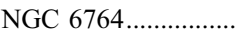 & 20 & 780 & $1.39 \times 1.26$ & 135 & 53 & 0.101 & $\ldots$ & 22.2 & 12 & $\mathrm{C}, \mathrm{L}$ \\
\hline 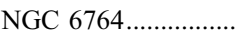 & 3.5 & 1040 & $2.50 \times 2.00$ & 16 & 54 & 0.029 & $\ldots$ & 21.7 & 24 & $\mathrm{C}, \mathrm{L}$ \\
\hline NGC $7469 \ldots \ldots \ldots \ldots \ldots$ & 20 & 1080 & $1.40 \times 1.29$ & -46 & 211 & 0.181 & 0.192 & 23.0 & 18 & $\mathrm{C}, \mathrm{E}$ \\
\hline 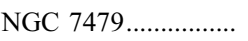 & 20 & 610 & $1.38 \times 1.32$ & -32 & 29 & 0.005 & 0.100 & 20.8 & 50 & $\mathrm{C}, \mathrm{L}$ \\
\hline 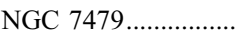 & 6 & 1590 & $1.21 \times 1.17$ & 56 & 21 & 0.006 & 0.041 & 20.9 & 39 & $\mathrm{C}, \mathrm{L}$ \\
\hline 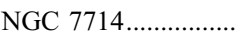 & 20 & 4770 & $1.38 \times 1.30$ & -2 & 83 & 0.0153 & 0.047 & 21.4 & 29 & $\mathrm{C}, \mathrm{E}$ \\
\hline 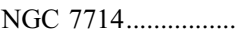 & 6 & 4340 & $1.28 \times 1.26$ & 58 & 95 & 0.0141 & $\ldots$ & 21.4 & 30 & $\mathrm{C}, \mathrm{E}$ \\
\hline 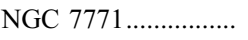 & 20 & 1040 & $1.33 \times 1.24$ & -45 & 38 & 0.0252 & 0.128 & 22.0 & 6 & $\mathrm{R}, \mathrm{E}$ \\
\hline 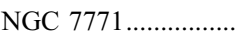 & 6 & 2270 & $1.16 \times 1.15$ & 0 & 21 & 0.0263 & 0.047 & 22.0 & 8 & $\mathrm{R}, \mathrm{E}$ \\
\hline
\end{tabular}

Notes. - The uncertainties in the total VLA fluxes are between 1 and $6 \mathrm{mJy}$. The uncertainties in the radio powers are less than $0.1 \mathrm{~W} \mathrm{~Hz}^{-1}$. Symbols for the

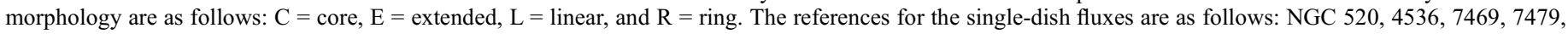

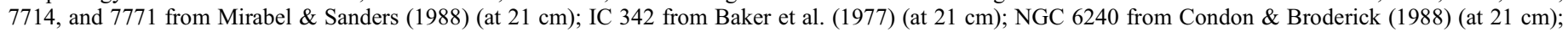
NGC 7771 from Sramek (1975) (at $6 \mathrm{~cm}$ ); and NGC 7479 from Condon et al. (1991) (at $6 \mathrm{~cm}$ ).

${ }^{a}$ Ratio of unresolved to resolved emission flux densities. The nuclear source was fitted with a Gaussian, and if its FWHM values were practically identical to the FWHM values of the beam, the nuclear source was called "unresolved."

major axis of the galaxy, implying that they are most likely emission coming from synchrotron radiation from electrons spiraling in the magnetic fields of the disk. For NGC 7469 and NGC 7714 the orientation of the radio continuum emission was very difficult to determine, and therefore, the discrepant values for these galaxies imply that there is only a more or less circular, extended nuclear component. The discrepant value for NGC 6240 is due to the peculiar western radio continuum loop, discussed in detail by Colbert et al. (1994). In NGC 6764 the almost perpendicular radio continuum emission suggests an outflow. The outflow will be discussed in more detail in another paper (S. Leon et al. 2006, in preparation). The Seyfert systems in our sample have an average difference of $60^{\circ}$ in the orientation of their radio continuum emission with respect to the position angle of the galaxy major axis. On the other hand, for the starburst galaxies the average value for the difference is $18^{\circ}$, or only $8^{\circ}$ if we exclude the uncertain case of NGC 7714. Such a difference between Seyfert and starburst galaxies could be explained by the existence of out-of-plane radio jets and outflows that take place preferentially in Seyfert galaxies. Our sample is too small to meaningfully compare the results to those of Schmitt et al. (1997), who found that the nuclear radio continuum structures in Seyfert 2 galaxies appear to have a fairly arbitrary orientation with respect to the galaxy major axis.

In general, the radial radio and NIR line emission profiles follow each other (Fig. 2). Exceptions are IC 342, in which the high surface brightness NIR line emission extends to larger radii than the radio emission, NGC 6574, in which Br $\gamma$ emission has a peak that is not seen in the other three profiles at radii around $10^{\prime \prime}$ from the center, and NGC 7771, in which the $\mathrm{Br} \gamma$ profile rises from the nucleus within $2^{\prime \prime}$, indicating the location of the starburst ring, while the other profiles are either constant or falling. The differences in the radio continuum morphology and NIR line emission morphology are likely due to the varying spatial dis- tribution of dust absorption (NIR lines) and magnetic fields (radio continuum). The radio profiles for galaxies that had radio observations at two wavelengths also follow each other well, except for NGC 6574. For NGC 6574 the starburst ring is more visible at $3.5 \mathrm{~cm}$. No systematic differences are seen between the profile trends and the relative radial displacement of the various emissions between Seyfert and starburst galaxies.

The size scale of the radio continuum emission in kiloparsecs is also listed in Table 4. This was taken as the major axis length of the FWHM of the two-dimensional Gaussian fit to the $20 \mathrm{~cm}$ emission in an image smoothed to $10^{\prime \prime}$ resolution, except for galaxies that essentially only have a nuclear component and little extended emission. For these and for NGC 6574, in which the extended emission is well separated from the nuclear emission, the $4 \sigma$ contours in Figure 1 were used. The average scale size of the radio continuum emission in Seyfert galaxies is $3.4 \mathrm{kpc}(\mathrm{rms}$ $1.1 \mathrm{kpc})$, and in starburst galaxies it is $1.5 \mathrm{kpc}(\mathrm{rms} 1.1 \mathrm{kpc})$. However, two of the nearest galaxies are in the starburst sample, and the lack of information in our observations at large scales (resolved out by the interferometer) does not warrant the conclusion that the size scales of circumnuclear radio continuum emission are different between the Seyfert and starburst galaxies. However, this is an interesting result that warrants follow-up studies using larger samples selected to have galaxies at comparable distances.

In Table 1 we list the deprojected bar lengths and ellipticities for our sample galaxies when a bar was detected. The deprojection method was identical to that used by Laine et al. (2002). We note that the galaxies with ringlike morphologies (except the peculiar case of NGC 6240; see below) all have bars. It has been established quite well that bars create resonance rings in the circumnuclear area (e.g., Sellwood \& Wilkinson 1993 and references therein). The connection between nuclear activity, bar properties, and circumnuclear rings is less well established. Within our 

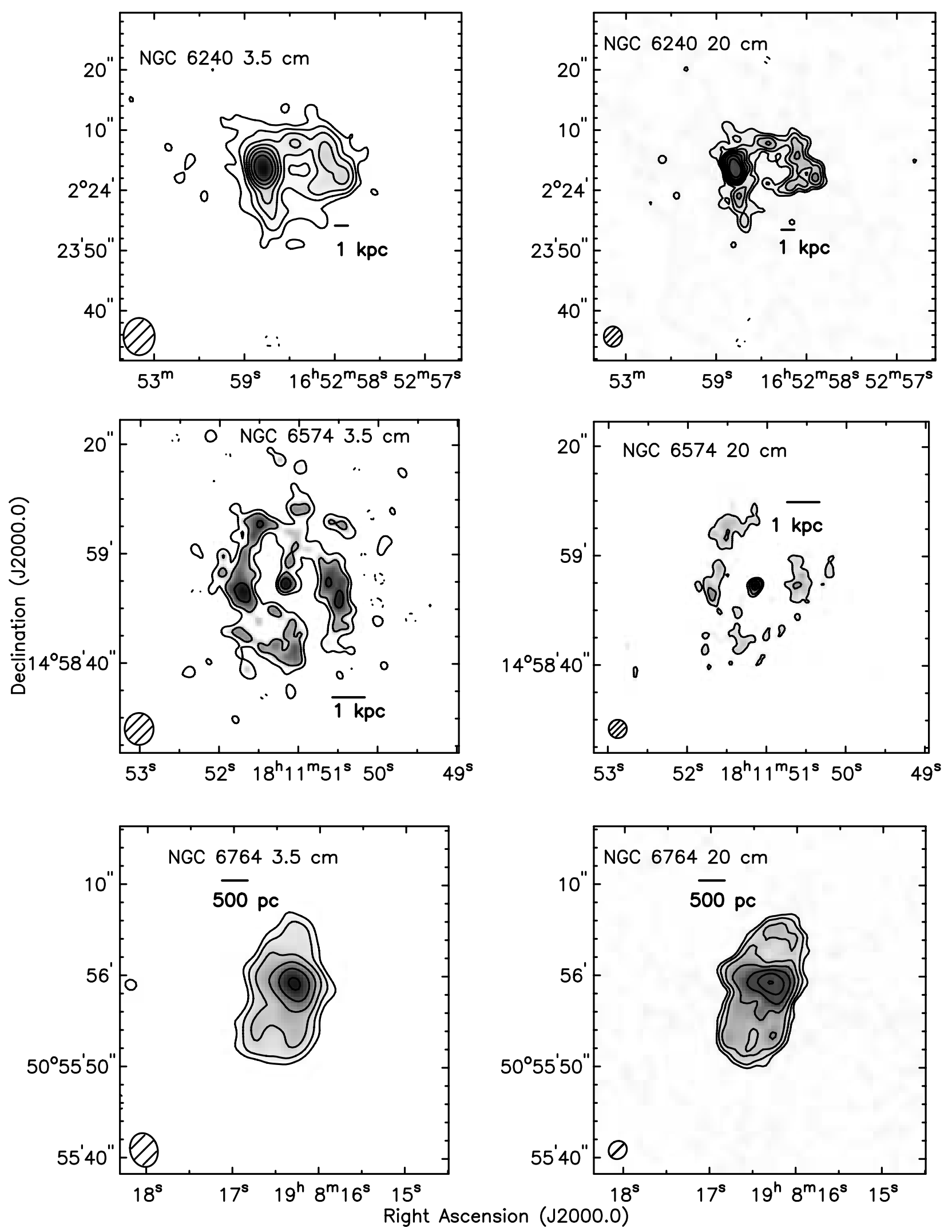

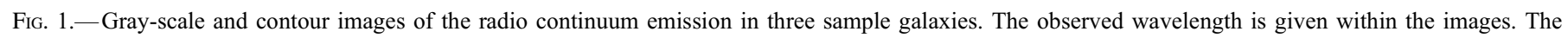

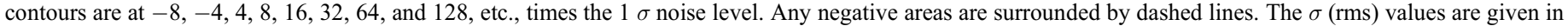
Table 3 . The beam size is indicated by the ellipse in the lower left corner of each image. The size scale is shown by the horizontal bar. 

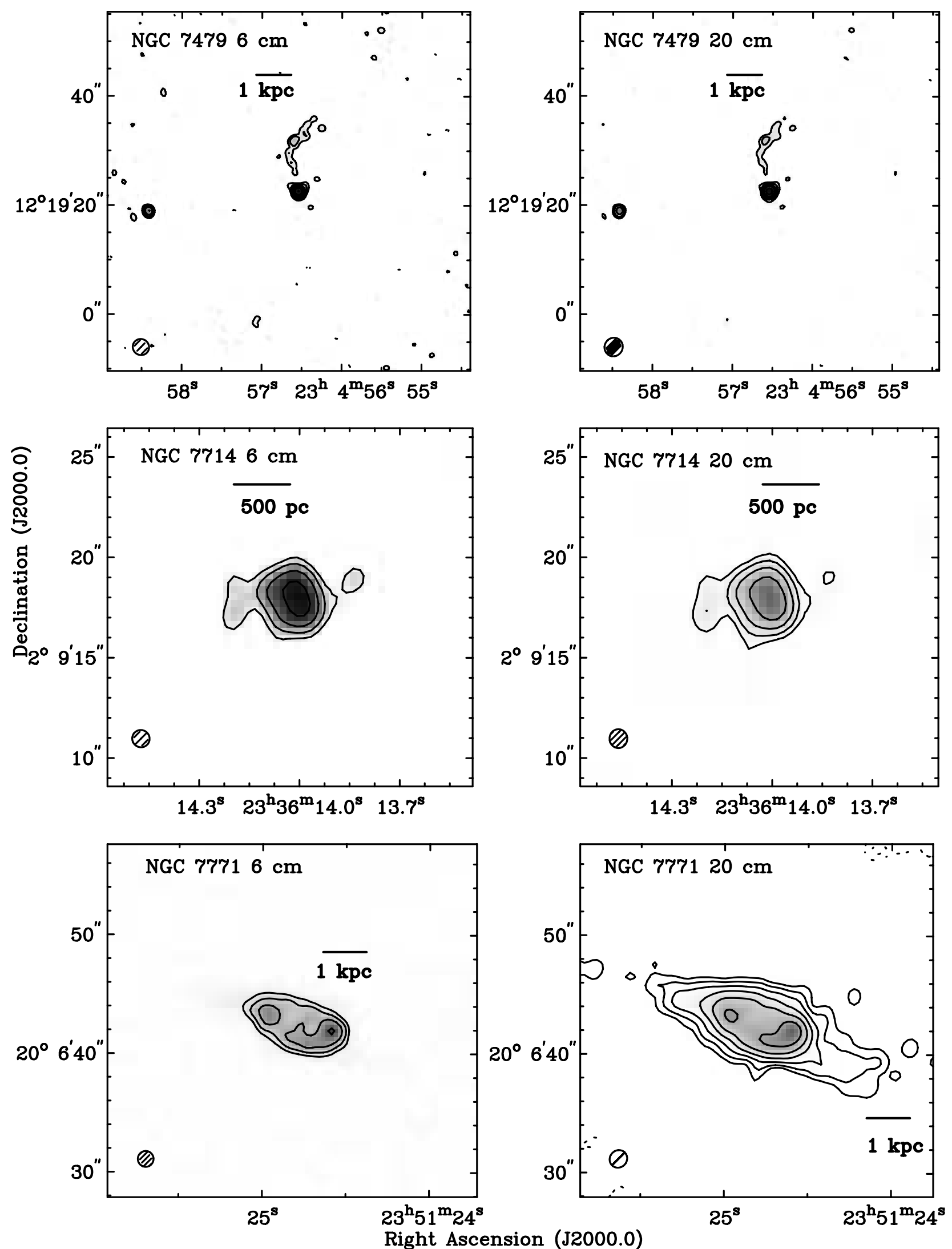

FIg. 1.-Continued 

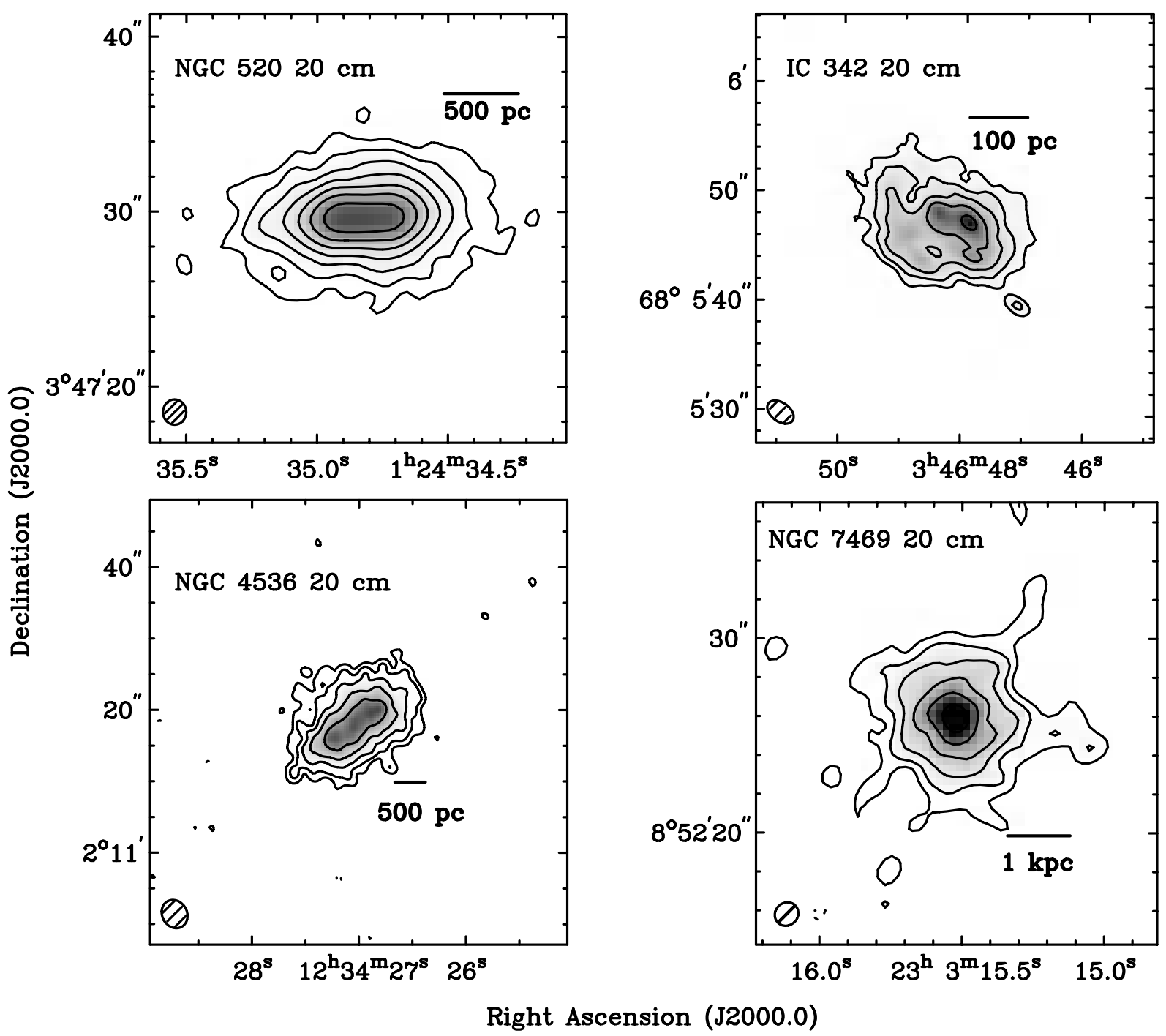

Fig. 1.-Continued

small sample, four galaxies have long and strong (high-ellipticity) bars (NGC 6764, NGC 7479, NGC 7714, and NGC 7771). Two of these are Seyfert galaxies and two are starburst galaxies. Four of our sample galaxies have short and weak bars (IC 342, NGC 4536, NGC 6574, and NGC 7469). Again, two of these are Seyfert galaxies and two are starburst galaxies. All the tabulated types of radio continuum morphologies are found among the galaxies with long and strong bars (centrally peaked, NGC 7714; linear "jetlike," NGC 7479; ringlike, NGC 7771; and outflow, NGC 6764). Ring, linear, and centrally peaked radio continuum morphologies are also found among the galaxies with short and weak bars.

The distance-independent radio powers at $20 \mathrm{~cm}$ are listed in Table 3. One should note that these powers are measured within widely varying physical sizes in the galaxies (from $\sim 400 \mathrm{pc}$ in IC 342 to $\sim 9.8 \mathrm{kpc}$ in NGC 6240; see Table 4). It appears that the Seyfert galaxies are slightly more powerful than the starburst galaxies in radio wavelengths. The merging system NGC 6240 has the greatest radio power. There also appears to be a slight tendency toward higher radio powers among the more strongly barred galaxies. This is in agreement with the result of Beck et al. (2002), who found that the average surface brightness in radio continuum correlates with the relative bar length.
Table 3 also includes an attempt to estimate the fraction of the radio continuum emission coming from the nuclear unresolved component after fitting a Gaussian to estimate its flux density. In all cases the extended emission dominates. Three out of five non-Seyfert galaxies have no unresolved component, while the average fraction of flux contributed by the unresolved component is about $25 \%$ for the Seyfert galaxies.

Finally, Table 5 shows the CAS (concentration, asymmetry, and clumpiness) measurements at $20 \mathrm{~cm}$ for all the sample galaxies. These measurements were made in a way similar to that in Conselice (2003). Concentration was measured in $3^{\prime \prime}$ and $10^{\prime \prime}$ radius apertures. In cases in which the central structure is clearly elliptical (NGC 520, NGC 4536, and NGC 7771) the measurements were made in elliptical annuli aligned along the major axis of the structure and having major axis lengths of $3^{\prime \prime}$ and $10^{\prime \prime}$. Naturally, since the distances of the galaxies vary by a large factor, these apertures measure different physical scales, but we consider it more important to measure the concentration regardless of the physical scale, since in almost all galaxies most of the detected radio continuum emission lies in scales of $10^{\prime \prime}-20^{\prime \prime}$ or less. The galaxies with the highest concentration indices are NGC 7771 , NGC 6240, and NGC 7469, as is also seen in the radio continuum emission figures. The asymmetry was calculated by 

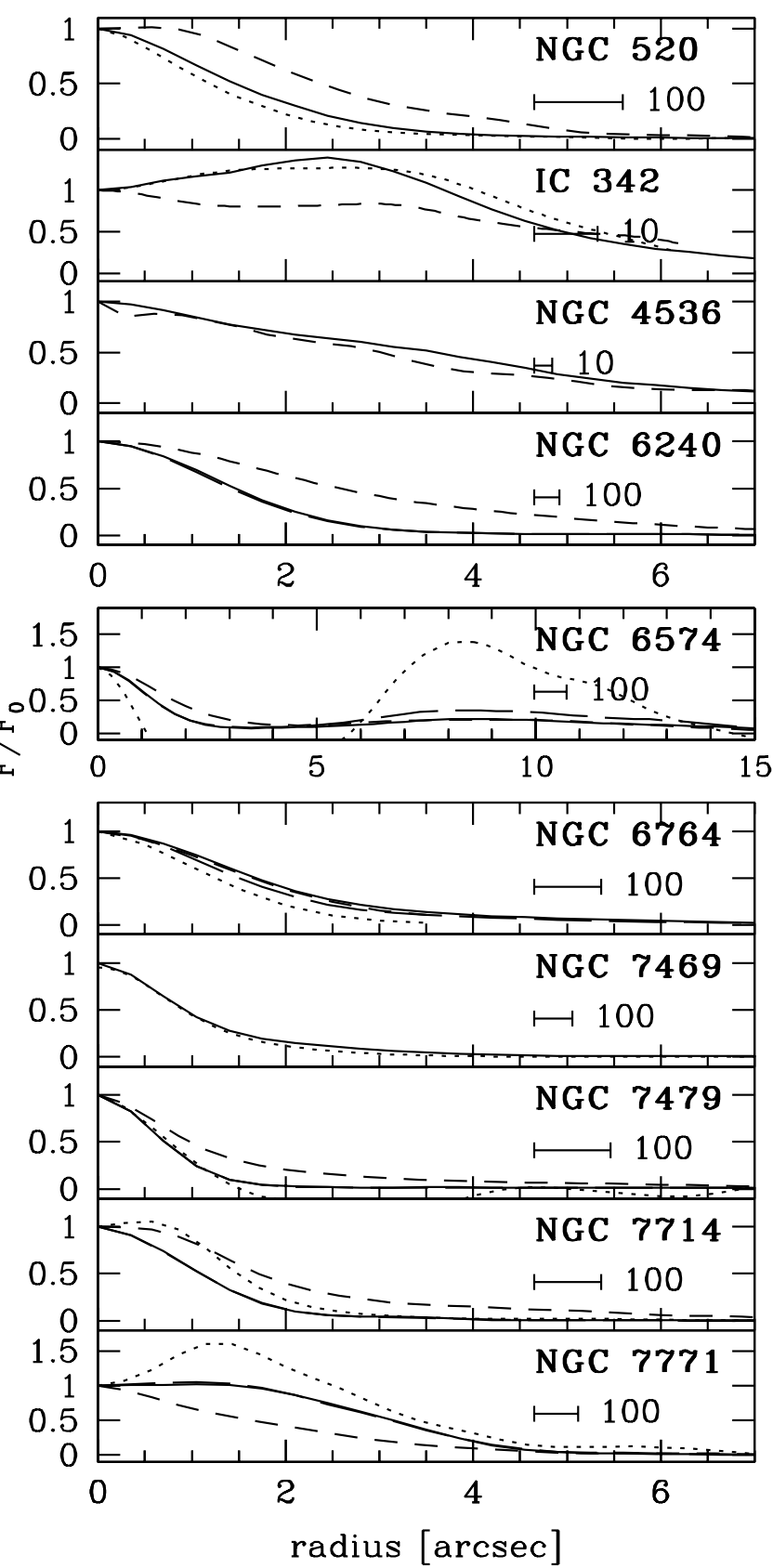

FIG. 2.-Radial surface brightness profiles for the 10 sample galaxies. The $20 \mathrm{~cm}$ radio continuum profile is shown by the solid lines, the $3.5 / 6 \mathrm{~cm}$ profiles by the long-dashed lines, the $\mathrm{H}_{2} 1-0 \mathrm{~S}(1)$ emission by the short-dashed lines, and the $\mathrm{Br} \gamma$ emission profile by the dotted lines. The profiles are normalized to the same relative intensity close to the center. The scale in parsecs (10 or 100) is also indicated.

rotating the image by $180^{\circ}$, then subtracting the rotated image from the original, taking the absolute value of the difference, and dividing the result by the original image. In addition, we only considered regions that had emission at higher than a $4 \sigma$ level in the original image (which was thus used as a mask). If a galaxy was perfectly symmetric, then the asymmetry index would have a value of zero. High values indicate a large degree of asymmetry. In NGC 7469 we used $8 \sigma$ as the threshold for masking. The most asymmetric galaxy is NGC 6240 with its one-sided emission structure. Clumpiness was calculated by subtracting an image smoothed to $5^{\prime \prime}$ resolution from the original image, then dividing the result by the original image. Thus, clumpy galaxies

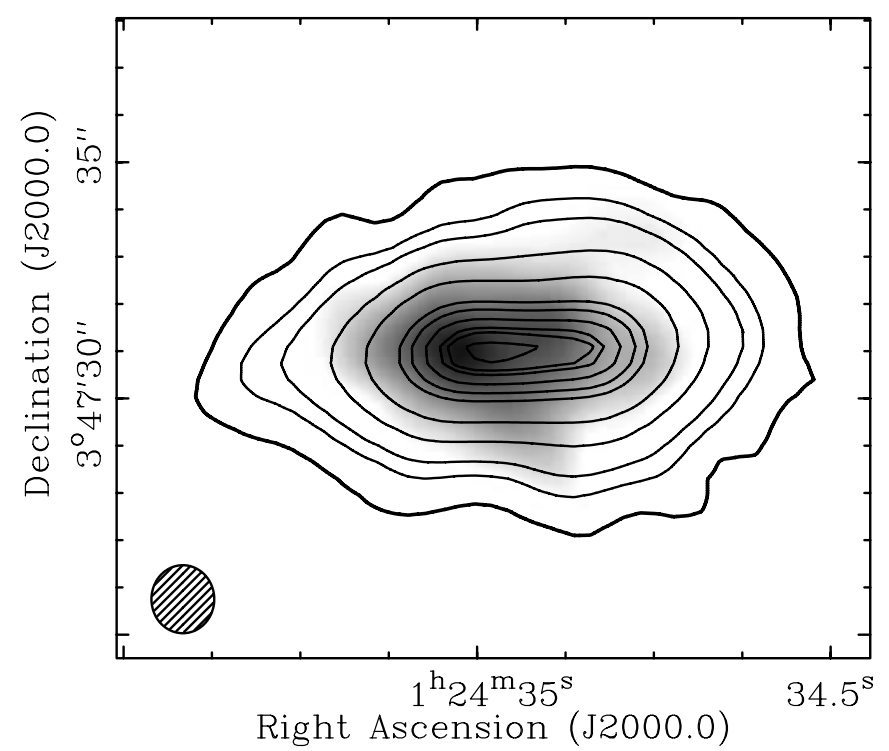

FIG. 3.- Gray-scale image of the $\mathrm{Br} \gamma$ emission with $20 \mathrm{~cm}$ radio continuum contours overlaid in NGC 520. The contour levels are at 8, 16, 24, 48, 96, 192, $240,300,356,380,420$, and 450 times the $1 \sigma$ noise level.

will have large clumpiness index values. The uncertainties in the measured quantities are estimated to be $<0.15$ for asymmetry, $<0.1$ for clumpiness, and $<0.05$ for concentration.

The average asymmetry, clumpiness, and concentration are not statistically different between Seyfert and non-Seyfert galaxies (Table 5). Therefore, we do not find any differences in the circumnuclear CAS properties in our sample of Seyfert and starburst galaxies.

\subsection{Star Formation and Radio Emission}

We searched the literature for SF and SN rates in the central regions of our sample galaxies. The results are displayed in Table 6. The given numbers have been adjusted to our adopted distances

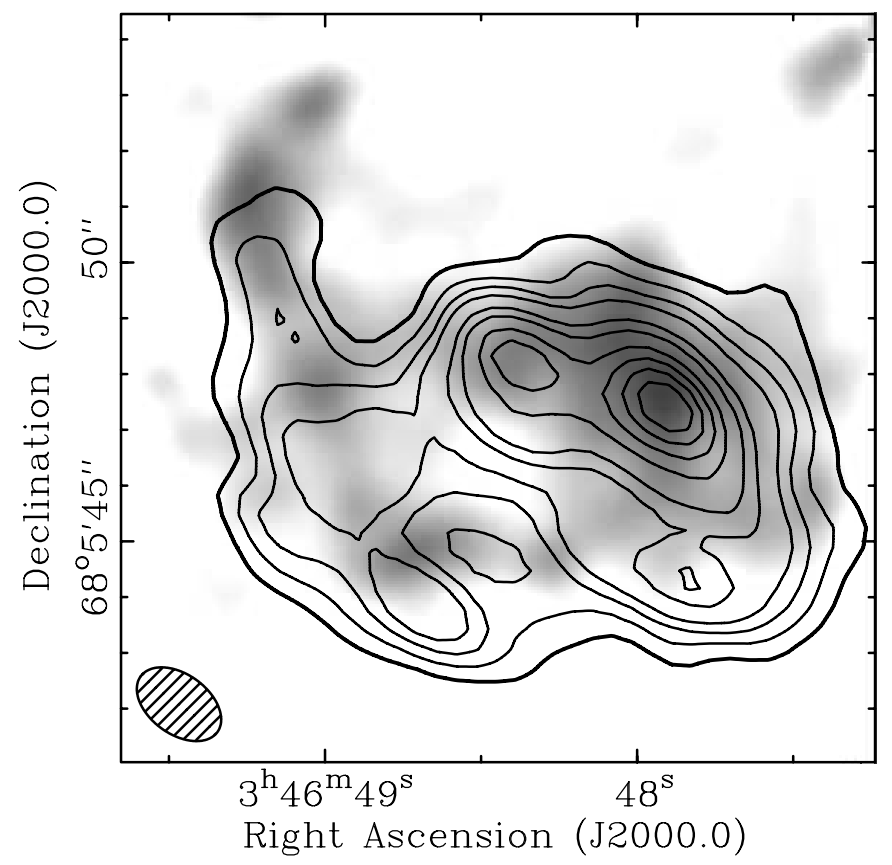

FIG. 4.-Gray-scale image of the $\mathrm{H}_{2} 1-0 \mathrm{~S}(1)$ emission with $20 \mathrm{~cm}$ radio continuum contours overlaid in IC 342 . The contour levels are at 12, 16, 20,24, $32,38,48,56,64,72$, and 88 times the $1 \sigma$ noise level. 


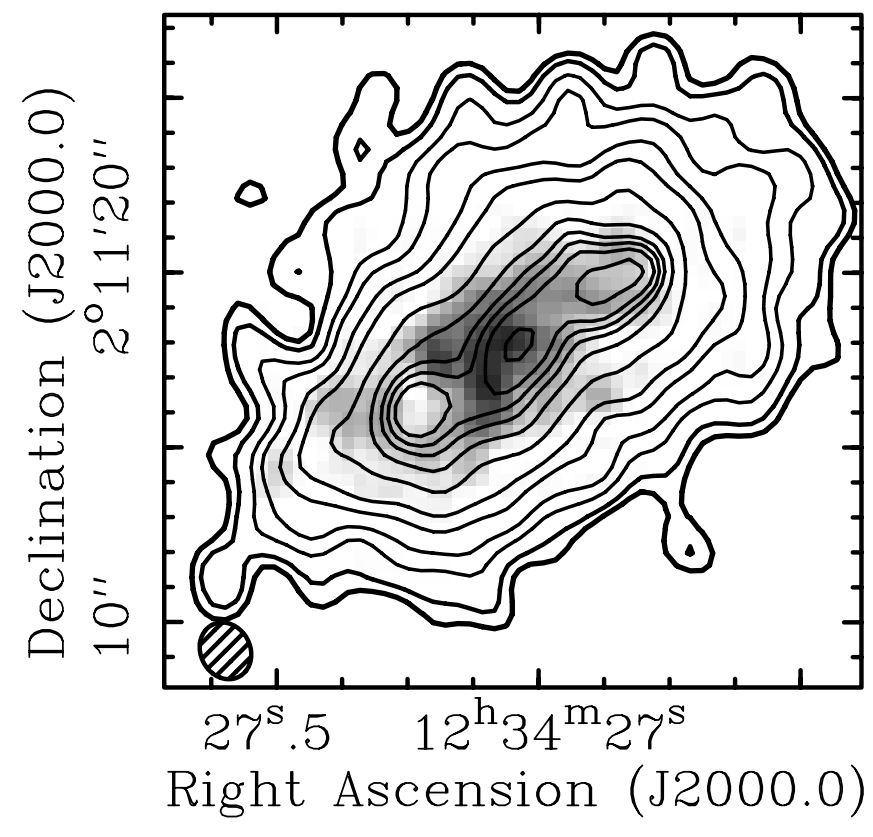

FIG. 5.- Gray-scale image of the $\mathrm{H}_{2} 1-0 S(1)$ emission with $20 \mathrm{~cm}$ radio continuum contours overlaid in NGC 4536. The contour levels are at $6,8,12$, $16,24,32,48,64,76,84,92$, and 104 times the $1 \sigma$ noise level.

as given in Table 1. Unfortunately, it was not possible to find the $\mathrm{SF}$ and $\mathrm{SN}$ information for all the sample galaxies. The SN rate was estimated from $\mathrm{Br} \gamma$ fluxes together with starburst models, or from radio continuum (usually at $20 \mathrm{~cm}$ ). Since there exists a relationship between $\mathrm{Br} \gamma$ and nonthermal radio continuum fluxes, the two methods are tied to each other. We expect Type II $\mathrm{SNe}$ to be the main contributors to the cosmic rays emitting the observed radio continuum in the circumnuclear starburst regions (e.g., Pannuti 2000). Note that the nonthermal radio continuum emission intensity strongly depends on the strength of the magnetic field, which may also be enhanced in the starburst regions (Beck et al. 2005). The SF rates in our sample galaxies vary

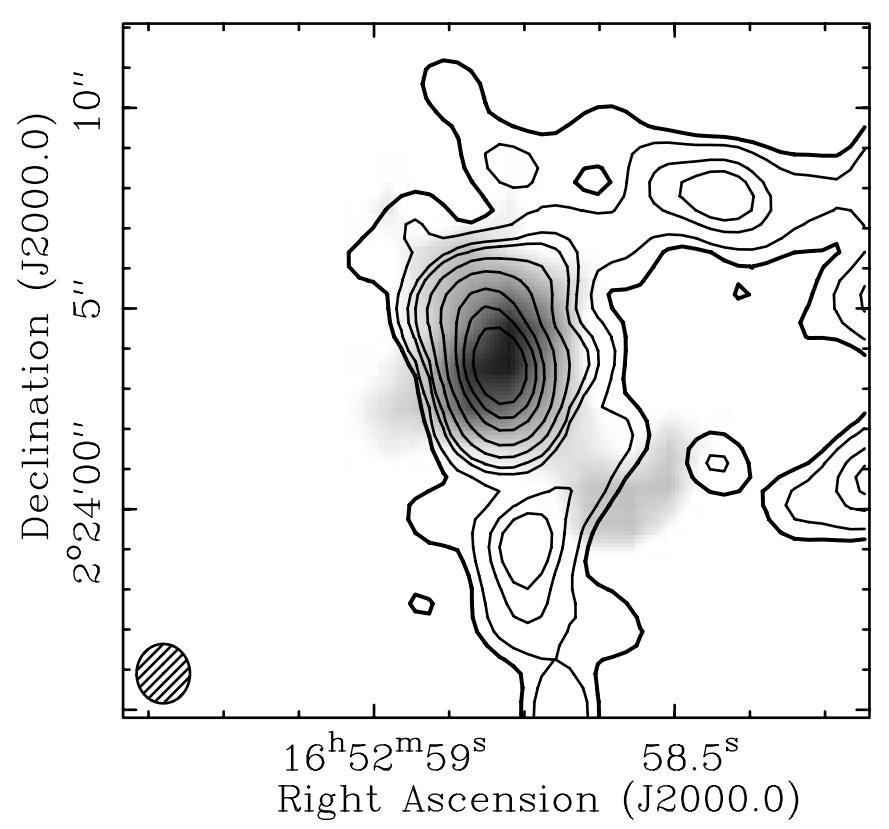

FIG. 6.- Gray-scale image of the $\mathrm{H}_{2} 1-0 S(1)$ emission with $20 \mathrm{~cm}$ radio continuum contours overlaid in NGC 6240. The contour levels are at 5, 8, 16, $24,48,96,192,300$, and 450 times the $1 \sigma$ noise level.

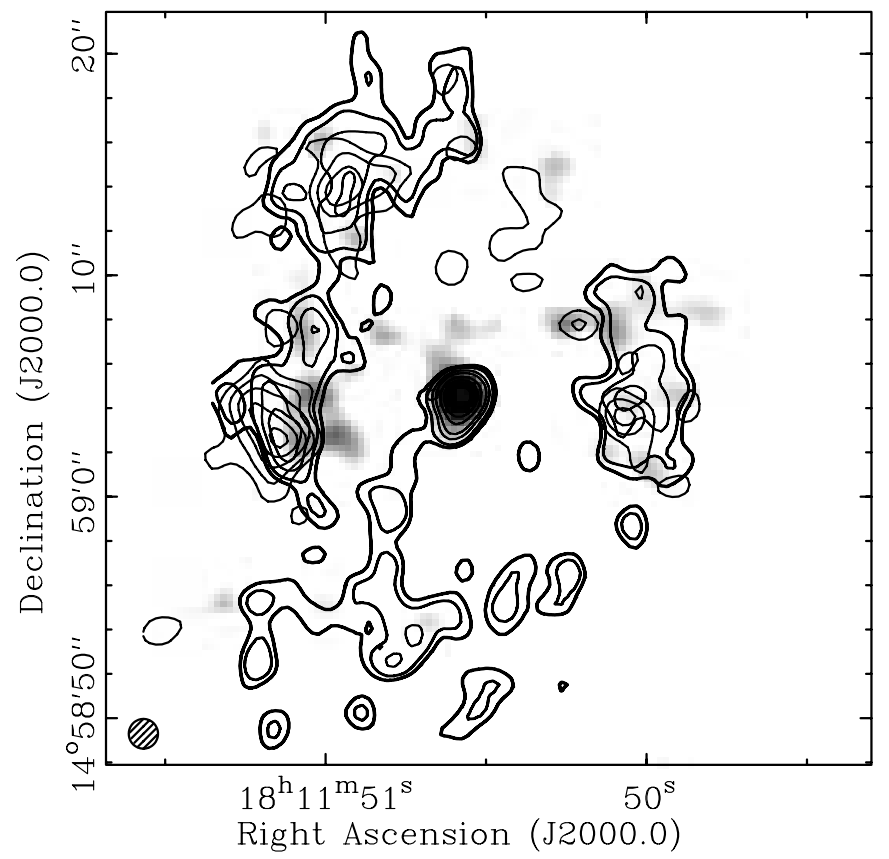

FIG. 7.-Gray-scale image of the $\mathrm{H}_{2} 1-0 \mathrm{~S}(1)$ emission with $20 \mathrm{~cm}$ radio continuum (black) and $\mathrm{Br} \gamma$ (light gray) contours overlaid in NGC 6574. The radio continuum contour levels are at $3,4,6,8,12,16$, and 24 times the $1 \sigma$ noise level.

between quiescent SF (a few tenths of solar masses per year) to several solar masses per year. There are no obvious differences between the SF rates of Seyfert and starburst galaxies. It is also well known that many Seyfert 2 galaxies have associated circumnuclear starbursts (e.g., Pogge 1989). Most of the SN rates are between 0.01 and $0.15 \mathrm{yr}^{-1}$, and there is no obvious difference between Seyfert and starburst galaxies. The highest $\mathrm{SN}$ rate, perhaps not surprisingly, is in the merging system NGC 6240.

We also studied the morphology and location of SF, traced mostly by $\mathrm{Br} \gamma$ emission, with respect to the radio continuum emission. The results are again given in Table 6. No clear trend can be seen. While the SF is coincident with the radio continuum

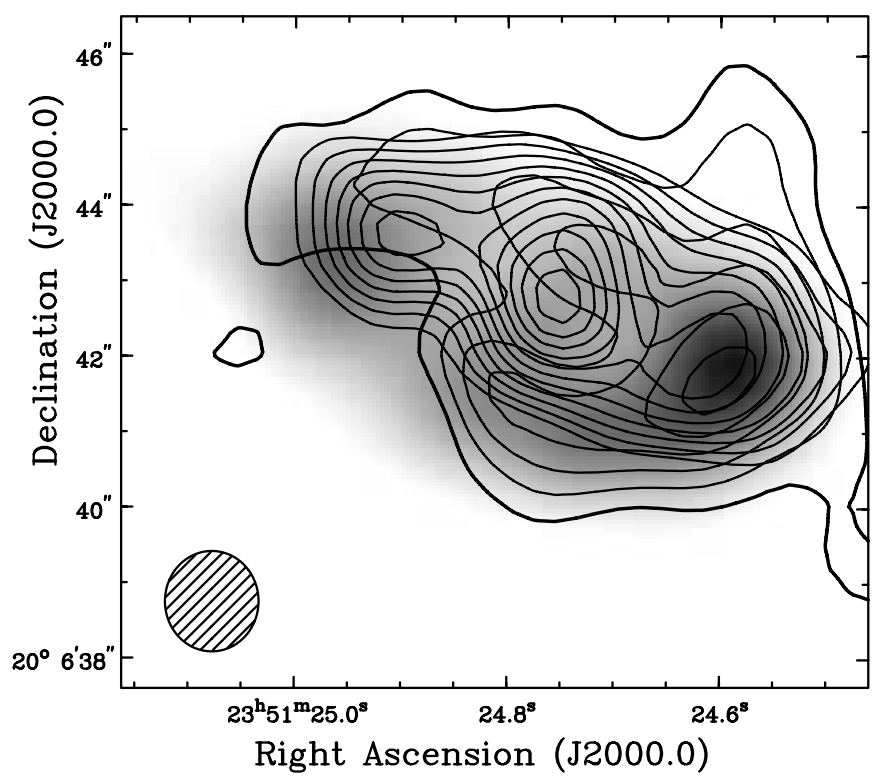

FIG. 8. - Gray-scale image of the $6 \mathrm{~cm}$ radio continuum emission with $\mathrm{Br} \gamma$ (black) and $\mathrm{H}_{2}$ 1-0 S(1) (dark gray) contours overlaid in NGC 7771 . 
TABLE 4

Orientation and Size Scale of Structures

\begin{tabular}{|c|c|c|c|c|c|}
\hline Galaxy & $\begin{array}{c}\text { Orientation of } \\
\text { Radio Continuum } \\
\text { (deg) }\end{array}$ & $\begin{array}{l}\text { Galaxy P.A. } \\
\text { (deg) }\end{array}$ & $\begin{array}{l}\text { Difference } \\
\text { (deg) }\end{array}$ & $\begin{array}{l}\text { Major-Axis Size } \\
\quad(\mathrm{kpc})\end{array}$ & $\begin{array}{l}\text { Inclination } \\
\text { (deg) }\end{array}$ \\
\hline NGC 520....................... & 97 & 93 & 4 & 1.6 & 70 \\
\hline IC $342 \ldots \ldots \ldots \ldots$ & 63 & 40 & 23 & 0.3 & 31 \\
\hline NGC 4536................... & 128 & 130 & 2 & 0.9 & 66 \\
\hline 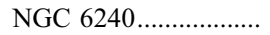 & 95 & 20 & 75 & 5.1 & 70 \\
\hline NGC $6574 \ldots \ldots \ldots \ldots \ldots$ & 10 & 160 & 30 & 4.1 & 37 \\
\hline 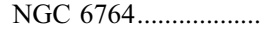 & 161 & 62 & 81 & 2.2 & 62 \\
\hline NGC 7469................... & 28 & 125 & 83 & 2.9 & 47 \\
\hline NGC $7479 \ldots \ldots \ldots \ldots$ & 170 & 22 & 32 & 2.5 & 51 \\
\hline 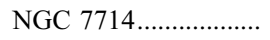 & 126 & 68 & 58 & 1.1 & 52 \\
\hline NGC $7771 \ldots \ldots \ldots \ldots \ldots . . . .$. & 69 & 68 & 1 & 3.6 & 75 \\
\hline
\end{tabular}

Notes.-The galaxy position angle of NGC 520 is deduced only from the inner 12" (Kotilainen et al. 2001). The galaxy position angle for IC 342 is deduced from the $\mathrm{H}$ I and CO observations of Crosthwaite et al. (2000, 2001). The galaxy position angles of NGC 4536, 6240, 6574, 6764, 7469, 7714, and 7771 have been taken from the Third Reference Catalogue of Bright Galaxies (de Vaucouleurs et al. 1991). The galaxy position angle of NGC 7479 has been taken from Laine \& Gottesman (1998). Inclination references are as follows: NGC 520, Yun \& Hibbard (2001); IC 342, Crosthwaite et al. (2000); NGC 4536, Jogee et al. (2005); NGC 6240, Tacconi et al. (1999); NGC 6574, Sakamoto et al. (1999); NGC 6764, Grosbøl (1985); NGC 7469, Genzel et al. (1995); NGC 7479, Laine \& Gottesman (1998); NGC 7714, Grosbøl (1985); NGC 7771, Nordgren et al. (1997).

emission in a few galaxies, there are SF features seen in only one image and not the other. This again suggests that radio continuum, especially at 6 and $20 \mathrm{~cm}$, is not a reliable tracer of the location of SF activity, but it traces the overall magnetic fields of the galaxy in which electrons released in $\mathrm{SN}$ events spiral and produce synchrotron radiation (Beck et al. 2005). Magnetic fields are only important if the nonthermal fraction of the emission is significant. Below we have estimated the contribution of the thermal emission for the galaxies that had $3.5 \mathrm{~cm}$ observations.

\subsection{Notes on Individual Galaxies}

\subsubsection{NGC 520}

NGC 520 is a merger system, presumably in an intermediate stage of merging, according to Toomre (1977). The primary nucleus (the southeastern nucleus) lies behind an intricate dust lane (Laine et al. 2003), and the optical, including $\mathrm{H} \alpha$, emission is highly extinguished there (Bernlöhr 1993; Laine et al. 2003). However, the decreasing extinction in NIR allows the detection

TABLE 5

CAS Parameters of Galaxies

\begin{tabular}{|c|c|c|c|}
\hline Galaxy & Asymmetry & Clumpiness & Concentration \\
\hline NGC $520 \ldots$ & 0.37 & 0.42 & 0.59 \\
\hline 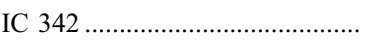 & 0.56 & 0.25 & 0.39 \\
\hline 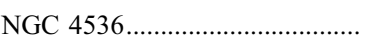 & 0.38 & 0.21 & 0.20 \\
\hline NGC $6240 \ldots \ldots \ldots \ldots \ldots \ldots \ldots$ & 0.90 & 0.38 & 0.73 \\
\hline NGC 6574 & 0.50 & 0.39 & 0.11 \\
\hline NGC $6764 \ldots \ldots .$. & 0.66 & 0.28 & 0.55 \\
\hline NGC $7469 . .$. & 0.27 & 0.30 & 0.69 \\
\hline NGC $7479 \ldots$ & 0.58 & 0.57 & 0.56 \\
\hline NGC $7714 \ldots \ldots$ & 0.27 & 0.47 & 0.81 \\
\hline NGC $7771 \ldots \ldots \ldots$ & 0.43 & 0.31 & 0.38 \\
\hline 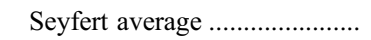 & $0.58 \pm 0.21$ & $0.38 \pm 0.10$ & $0.53 \pm 0.22$ \\
\hline Non-Seyfert average ................... & $0.40 \pm 0.09$ & $0.33 \pm 0.10$ & $0.47 \pm 0.21$ \\
\hline
\end{tabular}

NoTEs.-The asymmetry for NGC 7469 was calculated by masking out all the pixels below 8 times the $\sigma$ noise level in the original image. For other galaxies with more extended emission, 4 times the $\sigma$ noise level in the original image was used for masking. of $\mathrm{Br} \gamma$ emission in the nuclear area (Kotilainen et al. 2001). The radio continuum emission is much more extended than the $\mathrm{Br} \gamma$ emission, but the highest intensity, elongated radio continuum emission region coincides well with the $\operatorname{Br} \gamma$ emission (see Fig. 3). Since the origin of $\mathrm{Br} \gamma$ emission is $\mathrm{SF}$ and the $20 \mathrm{~cm}$ radio continuum is most likely nonthermal emission from electrons generated by $\mathrm{SNe}$ in starburst regions, this spatial coincidence is expected. The $\mathrm{H}_{2}$ emission is slightly more extended than the $\operatorname{Br} \gamma$ emission in NGC 520. There is no sign of circumnuclear ring structures either in radio continuum or in optical images. This is most likely due to the fact that the galaxy disk is highly inclined to the line of sight (Sanders et al. 1988). Our new data are more than a factor of 2 deeper than the Hummel et al. (1987) data taken in the same VLA configuration. The emission can be traced reliably within a $12^{\prime \prime} \times 8^{\prime \prime}$ area in our image, whereas the Hummel et al. (1987) map (only $6 \mathrm{~cm}$ shown, no $20 \mathrm{~cm}$ data shown) shows a structure only $6^{\prime \prime} \times 2$ ". 5 in extent. Our new $20 \mathrm{~cm}$ image shows more extended emission and hints at clumpy structure at lower emission levels not seen in the $20 \mathrm{~cm}$ VLA map published by Condon et al. (1990). Earlier subarcsecond-resolution VLA radio continuum observations at $2 \mathrm{~cm}$ (Carral et al. 1990), $6 \mathrm{~cm}$ (Condon et al. 1982), and $20 \mathrm{~cm}$ (Beswick et al. 2003) show a highly flattened and elongated multicomponent structure, corresponding to the highest intensity radio continuum structure seen in our $20 \mathrm{~cm}$ VLA image. The extended emission that we see in our map is resolved out in the Beswick et al. (2003) $20 \mathrm{~cm}$ map.

\subsubsection{IC 342}

IC 342 is the closest actively star-forming large spiral galaxy that has an almost face-on orientation to our line of sight. Bauer et al. (2003) suggest that the recent starburst of at least $0.5 M_{\odot}$ $\mathrm{yr}^{-1}$ in IC 342 was quite different from archetypal starbursts, since there is a lack of hot gas and luminous infrared emission. The nuclear cluster in IC 342 presumably formed in a starburst about 60 Myr ago (Böker et al. 1997, 1999). The registration of the radio continuum image with the $\mathrm{H}_{2} 1-0 S(1)$ line image (from Böker et al. 1997) in Figure 4 is uncertain because of the lack of absolute coordinate information in the latter image. We matched the position of the most intense emission on the northwestern side of the ring and checked that the northeastern emission feature 
TABLE 6

Star Formation and Supernova Rates

\begin{tabular}{|c|c|c|c|c|}
\hline Galaxy & $\begin{array}{l}\text { SF Rate } \\
\left(M_{\odot} \mathrm{yr}^{-1}\right)\end{array}$ & $\begin{array}{c}\text { SN Rate } \\
\left(\mathrm{yr}^{-1}\right)\end{array}$ & References & Relation of SF to Radio Continuum \\
\hline 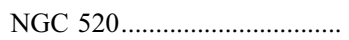 & 10 & 0.1 & 1 & Coincident \\
\hline IC $342 \ldots \ldots \ldots \ldots \ldots \ldots \ldots \ldots \ldots \ldots$ & 0.42 & 0.05 & 2,3 & \\
\hline 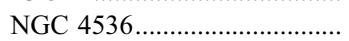 & $\ldots$ & 0.01 & 4 & Clumpier radio continuum \\
\hline 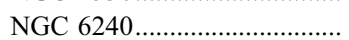 & $>278$ & 2.8 & 5,6 & Extra radio continuum loop \\
\hline NGC $6574 \ldots \ldots \ldots \ldots \ldots \ldots \ldots \ldots \ldots \ldots$ & 2.3 & 0.01 & 7 & $\mathrm{Br} \gamma$ "bar" not seen in radio continuum \\
\hline 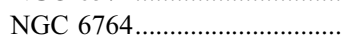 & 0.4 & 0.01 & 8 & Outflow seen in radio continuum and molecular gas \\
\hline NGC $7469 \ldots \ldots \ldots \ldots \ldots \ldots \ldots \ldots \ldots \ldots$ & 29 & 0.33 & 9,10 & $\ldots$ \\
\hline 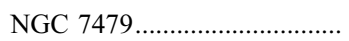 & 0.6 & $\ldots$ & 11 & Extra radio jet seen in radio continuum \\
\hline 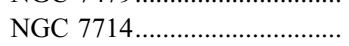 & 20 & 0.15 & 1 & Coincident \\
\hline NGC $7771 \ldots \ldots \ldots \ldots \ldots \ldots \ldots \ldots \ldots$ & 10 & 0.06 & 12 & $\ldots$ \\
\hline
\end{tabular}

Notes.-The second column gives the circumnuclear SF rate, and the third column gives the SN rate, with the references in the fourth column if available. The last column gives the morphological relation of SF, as seen in $\mathrm{Br} \gamma$ or $\mathrm{H} \alpha$ lines, to radio continuum.

References.-(1) Kotilainen et al. 2001; (2) Turner \& Hurt 1992; (3) Condon et al. 1982; (4) Davies et al. 1997; (5) Pasquali et al. 2004; (6) Colbert et al. 1994; (7) Kotilainen et al. 2000; (8) Schinnerer et al. 2000; (9) Genzel et al. 1995; (10) Smith et al. 1998; (11) Martin \& Friedli 1997; (12) Reunanen et al. 2000.

matches spatially. Assuming that the registration we used is correct, the ringlike structure in the radio continuum matches well with a similar structure seen in molecular hydrogen emission and also in other line images shown by Böker et al. (1997). Böker et al. (1997) explained the ring as the ILR ring of a weak stellar bar. A ringlike structure is also seen in optical Hubble Space Telescope (HST) V band (Böker et al. 1999) and archival $\mathrm{H} \alpha$ images, although the emission maxima in the optical seem to anticorrelate with the radio and NIR emission maps. This ringlike structure is bounded from outside by strong dust lanes, seen in optical HST images. The overall structure in our map is similar to the earlier $20 \mathrm{~cm}$ radio continuum map made at a comparable resolution by Condon et al. (1982) and the $6 \mathrm{~cm}$ map in Turner \& Ho (1983). However, our maps are more sensitive, and therefore, we are able to see fainter structures. Our new map has a higher $\mathrm{S} / \mathrm{N}$ than the 3 and $6 \mathrm{~cm}$ observations by Becklin et al. (1980).

\subsubsection{NGC 4536}

NGC 4536 is another nearby starburst galaxy. The molecular hydrogen 1-0 S(1) emission near the center was investigated by Davies et al. (1997; see also Fig. 5). They saw a strong NIR continuum peak in the nucleus, but the molecular hydrogen emission has peaks distributed in a pseudo-ring structure around the nucleus. Archival $H S T$ images taken near the $V$ and $H$ bands show that the radio continuum clumps are spatially correlated with SF regions in the spiral arms that are close to the nucleus in projection and not the nucleus itself. The radio continuum observations of Vila et al. (1990) at $6 \mathrm{~cm}$ show a similar overall structure, with peaks outside the nucleus, but their image has a much lower sensitivity than our new data. Our new VLA data at $20 \mathrm{~cm}$ are at least a factor of 3 deeper than the older Vila et al. (1990) data and show extended emission and three peaks lined up in the central region. Similarly, our observation is more sensitive than the $20 \mathrm{~cm}$ observation of Condon et al. (1982), allowing us to see fainter structures.

\subsection{4. $N G C 6240$}

NGC 6240 is a famous merging system in which two nuclei are close to merging together. The overall nuclear area of this system has LINER (Veilleux et al. 1995) and Seyfert 2 (e.g., Rafanelli et al. 1997) classifications. Two components are seen in subarcsecond-resolution radio continuum images (e.g., Carral et al. 1990; Beswick et al. 2001; Gallimore \& Beswick 2004), but our $1^{\prime \prime}-2^{\prime \prime}$ resolution data do not resolve the two merging nuclei either at 3.5 or at $20 \mathrm{~cm}$. In fact, Gallimore \& Beswick (2004) even see three radio components in VLBA observations of the nuclear region, two of which are likely to be Seyfert nuclei, based on their radio powers and brightness temperatures, and one that could be a radio SN. One of the Seyfert nuclei also has jetlike extensions. According to Lira et al. (2002) the AGN component has a bolometric luminosity of about $5 \times 10^{45} \mathrm{ergs} \mathrm{s}^{-1}$, making NGC 6240 a very powerful Seyfert galaxy. Only one peak is seen in the $\mathrm{H}_{2}$ line observations of van der Werf et al. (1993), who interpret the central peak to be located between the two nuclei as a result of a strong collision shock in the interstellar medium (see Fig. 6). A similar offset of the peak from the nuclei is seen in the maps of Tecza et al. (2000). The recent $\mathrm{H}_{2} 1-0 S(1)$ observations by Max et al. (2005) show a bridge of warm molecular gas between the northern and southern active nuclei. The [Fe II] $1.64 \mu \mathrm{m}$ and $\mathrm{Br} \gamma$ emission, on the other hand, coincide with the two nuclei (van der Werf et al. 1993; Tecza et al. 2000). The SF activity in the nuclear region was recently studied by Pasquali et al. (2004), who found extremely high SF and SN rates in the nucleus (see Table 6). Gerssen et al. (2004) studied the highresolution morphology using HST NICMOS and WFPC2 observations. They saw up to four nuclear components and a superwind from a nuclear starburst roughly in the east-west direction. Recent Chandra observations of the nucleus of NGC 6240 have revealed two main emission peaks, implying the existence of two supermassive black holes associated with the two merging nuclei (Komossa et al. 2003). The high SF and SN rates presumably drive a powerful superwind. The radio continuum emission in the nuclear region is most likely a combination of synchrotron emission from electrons created by the high SN rate and nonthermal emission from the active nuclei (Beswick et al. 2001). Scaling the $20 \mathrm{~cm}$ emission (assuming it is all nonthermal and using a spectral dependence of $S \propto \nu^{-0.6}, 0.6$ being a typical spectral index for the galaxies in our sample that had both 6 and $20 \mathrm{~cm}$ images) to $3.5 \mathrm{~cm}$ and subtracting it, we estimate that a considerable fraction of the emission at $3.5 \mathrm{~cm}$ can be thermal, up to $60 \%$. However, the spectral index of the nonthermal emission down to $3.5 \mathrm{~cm}$ is not known for NGC 6240 .

The most peculiar structure in this system is the extended western "arm" of radio continuum emission, which has several embedded peaks. This morphology has been interpreted as a result of synchrotron emission from a superwind, driven by the 
intense starburst in the nucleus (Colbert et al. 1994). Cosmic rays are generated in SF regions and propagate into the magnetic features. Our observation of the radio continuum morphology (the angle between the position angle of the underlying galaxy and the radio continuum emission) supports such a scenario. It is likely that the superwind seen in detail in the subarcsecond-resolution HST images of Gerssen et al. (2004) is related to the peculiar radio continuum structure. We note that NGC 6240 is the most radio powerful galaxy in our sample and has the highest SF and $\mathrm{SN}$ rates, together with nuclear activity. Our new $3.5 \mathrm{~cm}$ data have a similar resolution to the $3.5 \mathrm{~cm}$ data discussed by Colbert et al. (1994). We show our new data set for comparison with the old data. Antonucci (1985) published $6 \mathrm{~cm}$ data at a comparable resolution. Those data reveal a similar overall morphology as our map at $20 \mathrm{~cm}$ but at a much lower sensitivity.

\subsubsection{NGC 6574}

NGC 6574 is a nearby Seyfert 2 galaxy. It is the only galaxy in our sample that displays a central pointlike radio continuum component, a circumnuclear ring, and a connecting radio continuum feature between the two. Note that the ring was not detected in the early VLA high-resolution radio continuum observations of Vila et al. (1990), most likely because of inadequate sensitivity. Our new data are a factor of 5 more sensitive than the old data published by Vila et al. (1990). The "jet" is one-sided and is only at the $3 \sigma$ level in the $20 \mathrm{~cm}$ map (Fig. 7). At $3.5 \mathrm{~cm}$, where no previous high-resolution data exist to our knowledge, there is a connection between the nucleus and the circumnuclear ring on the opposite side of the nucleus at a $6 \sigma$ level. This "jet" has a major-axis radius of about $10^{\prime \prime}$ (just under $2 \mathrm{kpc}$ ). These "jets" lie close to the major axis of the galaxy and therefore are unlikely to represent any outflows perpendicular to the galaxy disk. Interestingly, there is moderate-strength $\mathrm{H}_{2}$ emission near the end of the southern "jet" (see Fig. 7). An estimate of the thermal emission in the $3.5 \mathrm{~cm}$ image, constructed in a similar way to that described above for NGC 6240, also shows that there is likely to be a substantial thermal component to the $3.5 \mathrm{~cm}$ emission in the ring north of the nucleus. The $\operatorname{Br} \gamma$ emission at this location is weak, so the northeastern radio continuum bridge is unlikely to be related to a "jet" striking the circumnuclear ring and triggering SF there. The nucleus seems to have only nonthermal radiation at $3.5 \mathrm{~cm}$, while elsewhere in the ring the thermal contribution could be up to $50 \%$ based on estimating the nonthermal contribution from the $20 \mathrm{~cm}$ image by scaling it down to $3.5 \mathrm{~cm}$ with a spectral index of 0.6 . There appears to be a nuclear stellar (and perhaps gaseous, as seen by Kotilainen et al. 2000) bar at a position angle of about $150^{\circ}$ inside the ring. The strongest radio continuum emission occurs in the ring east of the nucleus, as does the strongest $\mathrm{Br} \gamma$ and $\mathrm{H}_{2} 1-0 S(1)$ emission (see Fig. 7). There is a fairly good coincidence between the $\mathrm{H}_{2} 1-0$ $S(1)$ and radio continuum emission, although there is a region of moderately strong radio continuum emission south-southeast of the nucleus in the ring that does not show up in the NIR line emission. However, the absence of any detected $\mathrm{H}_{2}$ emission there is likely due to the narrow bandwidth of the Fabry-Perot interferometer filter used in the observations of Kotilainen et al. (2000). An archival HST WFPC2 ultraviolet image at $3000 \AA$ shows a patchy star-forming ring, but the detailed spatial correlation with the strongest radio and NIR line emission is poor within the ring.

\subsection{6. $N G C 6764$}

There is a remarkable outflow structure clearly revealed by our radio continuum images of NGC 6764 approximately per- pendicular to the galaxy major axis and therefore probably perpendicular to the plane of the galaxy (see Fig. 1). The only other earlier data at comparable resolution at $20 \mathrm{~cm}$ were published by Condon et al. (1982), but those data have a sensitivity that is a factor of 2 worse than in our new data. The 6 and $21 \mathrm{~cm}$ Westerbork maps by Baum et al. (1993) have a lower spatial resolution and sensitivity than our maps. Our map shows clear cavities in the radio continuum emission, which are not shown by the Condon et al. (1982) map. Our $3.5 \mathrm{~cm}$ map is the first high-resolution map showing the radio continuum morphology at that wavelength. Despite the outflow morphology, the SF and $\mathrm{SN}$ rates are not remarkably high, and therefore, it is possible that continuous SF models give too low SF rates and there actually was a starburst near the nucleus about 3-5 Myr ago, as suggested by Schinnerer et al. (2000), who also discuss the molecular gas outflow in this galaxy. There appears to be little thermal emission in NGC 6764 at $3.5 \mathrm{~cm}$ based on the scaling of the $20 \mathrm{~cm}$ emission with a spectral index of 0.6. Since this galaxy has a relatively strong stellar bar, there is a clearly identifiable mechanism to bring fuel to the central active region. $\mathrm{H}_{2} 1-0 S(1)$ and $\mathrm{Br} \gamma$ emission in NGC 6764 is relatively weak (J. Kotilainen et al. 1998, unpublished). The nucleus of NGC 6764 has the activity of a Seyfert 2 or LINER type and also has a clear starburst signature (Rubin et al. 1975; Gonçalves et al. 1999). The bolometric luminosity of the nucleus in NGC 6764 is about $5 \times$ $10^{43} \mathrm{ergs} \mathrm{s}^{-1}$ (Eckart et al. 1996), including both the starburst and the nonstellar component.

\subsection{7. $N G C 7469$}

NGC 7469 has a Seyfert 1.2 nucleus, and a starburst ring of $3 "$ diameter around the nucleus (Miles et al. 1996) is seen in a wellresolved WFPC2 image (Malkan et al. 1998). In radio continuum at $1^{\prime \prime}-2^{\prime \prime}$ resolution the nucleus shows up as an unresolved point source surrounded by diffuse emission (see Fig. 1). The ring is not resolved in our radio continuum images. Condon (1980) shows a $6 \mathrm{~cm}$ image but no $20 \mathrm{~cm}$ data. His $6 \mathrm{~cm}$ map suffers from a highly elongated beam. Ulvestad et al. (1981) show a $6 \mathrm{~cm}$ map at $1^{\prime \prime}-2^{\prime \prime}$ resolution, but it suffers from an elongated beam and shows suspicious features not seen in our new $20 \mathrm{~cm}$ map. Unger et al. (1987) show a $20 \mathrm{~cm}$ map at about 1 ."5-2" resolution from observations taken in 1983. Their map has a lower $\mathrm{S} / \mathrm{N}$ than our new map and a poorer spatial resolution, and their map does not show the extended emission as well as our new map. Subarcsecond-resolution radio continuum images by Wilson et al. (1991), Colina et al. (2001), Thean et al. (2001), and Lal et al. (2004) resolve the nuclear and ring components, but those maps do not show the extended emission seen in our new map. VLBI observations have shown that the nucleus actually consists of three emitting regions aligned in the east-west direction (Lonsdale et al. 2003). ROSAT observations (Perez-Olea $\&$ Colina 1996) also show centrally peaked emission, somewhat similar to the radio continuum morphology in our new image at $20 \mathrm{~cm}$. NIR line imaging observations show hints of the starburst ring at a radius of about 1".5 (Genzel et al. 1995). The radio power of NGC 7469 is among the highest in our sample, probably reflecting the powerful Seyfert 1 nucleus. The bolometric luminosity is about $8 \times 10^{44} \mathrm{ergs} \mathrm{s}^{-1}$ (Lonsdale et al. 2003). The SF rate in the nucleus, using continuous SF models, is also very high, around $30 M_{\odot} \mathrm{yr}^{-1}$ (Genzel et al. 1995).

\subsubsection{NGC 7479}

Neff \& Hutchings (1992) present a $20 \mathrm{~cm}$ image taken with VLA in the A configuration in 1989 and therefore at a resolution comparable to our new data. However, our new $20 \mathrm{~cm}$ map has a 
higher sensitivity due to VLA $L$-band sensitivity improvement in the early 1990 s by a factor of 2 . Ho \& Ulvestad (2001) published comparable-resolution maps at both 6 and $20 \mathrm{~cm}$. We choose to show the map from our observations, and it can be compared to that displayed in Figure 15 of Ho \& Ulvestad (2001). The jetlike structure of NGC 7479, seen in Figure 1, was noted by Laine \& Gottesman (1998) and is the subject of a detailed study with polarized radio continuum emission (R. Beck \& S. Laine 2006, in preparation). The jetlike feature probably has a magnetic origin. However, there is no clear evidence for jetlike features that would connect to the circumnuclear region and trigger activity there. The jetlike structure has a total extent of several kiloparsecs if it lies in the galaxy plane. However, it is more likely that it is an out-of-plane structure (R. Beck \& S. Laine 2006, in preparation). The radio continuum has a pointlike nucleus that is unresolved even in subarcsecond-resolution MERLIN radio continuum observations (S. Laine 1998, unpublished). The nucleus was classified as Seyfert 1.9 by Ho et al. (1997b) and as a LINER by Keel (1983). The unpublished NIR line observations show a nuclear disk (with a diameter of about $3^{\prime \prime}$ ) of $\mathrm{H}_{2} 1-0 S(1)$ emission. The center also has a molecular gas disk seen in $\mathrm{CO}$ observations (Laine et al. 1999), which peaks at the position of the radio continuum peak within the uncertainties. Archival HST NICMOS and WFPC2 images show a strong dust lane approaching from the northwest and continuing to within a few tens of parsecs from the moderately bright nucleus. No circumnuclear ring has been detected within the strong stellar bar. The SF estimate is uncertain due to the large amount of dust and gas near the nucleus.

\subsection{9. $N G C 7714$}

NGC 7714 is a nearby interacting starburst galaxy. There is a strong nuclear and circumnuclear starburst. The $\mathrm{Br} \gamma$ emission [and the $\mathrm{H}_{2} 1-0 S(1)$ emission] has an extension toward the eastsoutheast, also hinted at by our radio continuum images at 6 and $20 \mathrm{~cm}$ (see Fig. 1). This coincides with a spiral arm-like starforming feature seen in an HST WFPC2 F606W image (Smith et al. 1999, 2005). In addition to the NIR line features, our radio continuum images show a point source about $3^{\prime \prime}$ to the westnorthwest of the nuclear component, perhaps hinted at in the $\mathrm{H}_{2}$ 1-0 S(1) image of Kotilainen et al. (2001). Continuous SF models give a very high SF rate for NGC 7714, $20 M_{\odot} \mathrm{yr}^{-1}$ (Kotilainen et al. 2001). An earlier $6 \mathrm{~cm}$ image by Weedman et al. (1981) has a much lower $\mathrm{S} / \mathrm{N}$ and shows suspicious structures not seen in our new, higher sensitivity data. The $6 \mathrm{~cm}$ image in Stine (1992) does not show the same structures that are apparent in our new images due to a different contrast in gray-scale and contour spacing.

\subsubsection{0. $N G C 7771$}

NGC 7771 is another interacting starburst galaxy that has a clear circumnuclear starburst ring. However, this galaxy is highly inclined $\left(75^{\circ}\right)$ to the line of sight. The southwestern side of the ring has the strongest radio continuum emission at both 6 and $20 \mathrm{~cm}$, as well as in $\mathrm{Br} \gamma$ emission (Fig. 8; NIR data from Reunanen et al. 2000). Our data are a factor of 5 deeper than earlier $6 \mathrm{~cm}$ observations at comparable resolution by Batuski et al. (1992). Subarcsecond-resolution radio continuum observations have resolved the ring into numerous components, and they also reveal a nuclear radio continuum component (Neff \& Hutchings 1992). Note that the more extended emission that we see in our new data is resolved out in these higher resolution observations. The constant SF model would require very high SF rates, and therefore, it is more likely that the SF took place in a starburst 6-7 Myr ago
(Reunanen et al. 2000). A very recent starburst in this galaxy was also suggested by Smith et al. (1999), who performed a case study of SF in the circumnuclear area of this galaxy using new NIR data and existing high-resolution radio continuum images.

\section{SUMMARY}

Our sample of 10 starburst, Seyfert, and merging or interacting galaxies has revealed a mixed morphology in their circumnuclear regions. Ring, linear, and "jetlike" structures and nuclear point sources are seen in all three types of galaxies. We looked carefully for the connection between the nucleus and the surrounding region to obtain further clues about what makes galaxies active. Our results reinforce the notion that there is no unambiguous feature even in the radio continuum emission that would indicate how the nuclear activity and the surrounding $1 \mathrm{kpc}$ scale circumnuclear region are causally linked to each other, or how they would evolve together. The active nuclei do not reveal any jets or other channels by which they would trigger $\mathrm{SF}$ in the surrounding disk or ring. Nor does there seem to be any particular features in the circumnuclear rings or disks that could be used as indicators of nuclear activity. Studies at other wavelengths have also concluded that there are no obvious causal or spatial connections between nuclear activity and the properties of the circumnuclear region (Storchi-Bergmann et al. 2001; Martini et al. 2003; Márquez et al. 2004).

We have tabulated the radio powers in the circumnuclear region of our sample galaxies. Seyfert galaxies appear to have slightly larger average radio powers. In galaxies that have a core component, the ratio of the core component to circumnuclear extended radio continuum emission is typically between $30 \%$ and $40 \%$, independent of the activity or interaction class of the galaxy. The physical size of the circumnuclear radio emission region is typically $1-2 \mathrm{kpc}$, although there is a hint that Seyfert galaxies may have larger circumnuclear radio emission sizes than non-Seyfert galaxies. There is no correlation between the AGN bolometric luminosity and the radio morphology. For example, the Seyfert galaxies with the largest AGN luminosities in our sample (NGC 6240 and NGC 7469) exhibit different morphologies. NGC 6240 possesses small-scale jets and a nuclear outflow, which is likely connected to the peculiar western radio continuum loop. NGC 7469 has an aligned multicomponent radio continuum structure seen with VLBI observations, but its circumnuclear radio morphology seen in our 1".4 resolution observations does not present any specific structures, only a nuclear component with some diffuse emission around it. The AGNs with lower bolometric luminosities have a widely varying radio continuum structure as well. NGC 6574 has a circumnuclear radio continuum ring, NGC 6764 has an outflow structure in the radio continuum, and finally, NGC 7479 has peculiar large-scale radio "jets." A nuclear starburst exhibits itself in various ways in the radio continuum morphology. NGC 6764 has a clear outflow morphology, while NGC 6240 has a peculiar loop structure on one side of the nucleus, possibly as a result of a superwind from several SN explosions in the nucleus (Colbert et al. 1994). Perhaps the most intriguing structure is seen in NGC 7479, which has a jetlike morphology together with a core component but no extended nuclear starburst region. This galaxy has been speculated to be experiencing a minor merger (Laine \& Heller 1999). It also has a massive molecular disk around the nucleus (Laine et al. 1999). Future investigation is warranted into any connection between a minor merger, the nuclear activity, and the radio "jet."

We have compared the radial surface brightness profiles of radio emission and $\mathrm{Br} \gamma$ and/or $\mathrm{H}_{2}$ line emission. While the 
starburst rings show up clearly in the $\mathrm{Br} \gamma$ emission, we see no systematic differences between the Seyfert and starburst galaxies in these profiles. We also calculated the CAS parameters for our sample galaxies. Again, the average concentration, asymmetry, and clumpiness parameters are not significantly different between Seyfert and starburst galaxies. The orientation of the radio continuum emission differs from the orientation of the galaxy major axis more in Seyfert galaxies than in starburst galaxies. We have also used SF and SN rates found in the literature to estimate how they depend on the distribution of radio continuum emission and the activity class of the galaxy. We have inspected the correlation of the SF and SN rates with the strength of nonstellar nuclear activity or interaction class but found no clear trends.

Finally, we have compared the radio continuum morphology to that of NIR line emission morphologies, including $\mathrm{Br} \gamma$ and $\mathrm{H}_{2}$ line emission, when available. We find that the bulk of the radio continuum usually traces the sum of the $\mathrm{Br} \gamma$ and $\mathrm{H}_{2}$ line emission fairly reliably. However, several extranuclear radio continuum emission features not visible in the NIR line images were found, including the jetlike structures and the outflow-type structures. These differences are likely caused by the effects of dust absorption and scattering (affecting NIR emission) and the spatial distribution and strength of magnetic fields (affecting radio continuum emission).

Our results will be extended and validated in a follow-up study that will include a much larger sample of Seyfert and starburst galaxies. The information content of the radio contin- uum observations will be increased by including polarization information. Unfortunately, such observations are time-expensive, and the next step we intend to take includes a detailed study of the magnetic origin of the jetlike structure in NGC 7479.

We thank Torsten Böker, Richard Davies, and Paul van der Werffor providing us with NIR line images. We are also thankful to Ray Norris for comments on an earlier draft of this paper, to Mark Lacy for discussions on the radio jet-AGN luminosity correlation, and to Tom Pannuti for discussions on the radio continuum emission from supernova remnants. We are thankful to the referee, W. C. Keel, for suggestions that improved the quality of the paper. J. K. K. was supported by the Academy of Finland (project 8201017) during part of this work. The National Radio Astronomy Observatory is a facility of the National Science Foundation operated under cooperative agreement by Associated Universities, Inc. This research has made use of the NASA/IPAC Extragalactic Database (NED), which is operated by the Jet Propulsion Laboratory, California Institute of Technology, under contract with the National Aeronautics and Space Administration. This publication makes use of data products from the Two Micron All Sky Survey, which is a joint project of the University of Massachusetts and the Infrared Processing and Analysis Center, California Institute of Technology, funded by the National Aeronautics and Space Administration and the National Science Foundation.
Alonso-Herrero, A., Ryder, S. D., \& Knapen, J. H. 2001, MNRAS, 322, 757

Antonucci, R. R. J. 1985, ApJS, 59, 499

Athanassoula, E. 1992, MNRAS, 259, 345

Baker, J. R., Haslam, C. G. T., Jones, B. B., \& Wielebinski, R. 1977, A\&A, 59, 261

Barnes, J. E., \& Hernquist, L. E. 1991, ApJ, 370, L65

Barth, A. J., Ho, L. C., Filippenko, A. V., \& Sargent, W. L. 1995, AJ, 110, 1009

Batuski, D. J., Hanisch, R. J., \& Burns, J. O. 1992, AJ, 103, 1077

Bauer, F. E., Brandt, W. N., \& Lehmer, B. 2003, AJ, 126, 2797

Baum, S. A., O’Dea, C. P., Dallacassa, D., de Bruyn, A. G., \& Pedlar, A. 1993, ApJ, 419, 553

Beck, R., Ehle, M., Shoutenkov, V., Shukurov, A., \& Sokoloff, D. 1999, Nature, 397, 324

Beck, R., Fletcher, A., Shukurov, A., Snodin, A., Sokoloff, D. D., Ehle, M., Moss, D., \& Shoutenkov, V. 2005, A\&A, 444, 739

Beck, R., Shoutenkov, V., Ehle, M., Harnett, J. I., Haynes, R. F., Shukurov, A., Sokoloff, D. D., \& Thierbach, M. 2002, A\&A, 391, 83

Beck, S. C., Turner, J. L., \& Kovo, O. 2000, AJ, 120, 244

Becklin, E. E., Gatley, I., Matthews, K., Neugebauer, G., Sellgren, K., Werner, M. W., \& Wynn-Williams, C. G. 1980, ApJ, 236, 441

Benedict, G. F., Smith, B. J., \& Kenney, J. D. P. 1996, AJ, 111, 1861

Benedict, G. F., et al. 1993, AJ, 105, 1369

Bernlöhr, K. 1993, A\&A, 270, 20

Beswick, R. J., Pedlar, A., Clemens, M. S., \& Alexander, P. 2003, MNRAS, 346,424

Beswick, R. J., Pedlar, A., Mundell, C. G., \& Gallimore, J. F. 2001, MNRAS, 325,151

Bian, W.-H., \& Zhao, Y.-H. 2003, PASJ, 55, 599

Böker, T., Förster-Schreiber, N. M., \& Genzel, R. 1997, AJ, 114, 1883

Böker, T., van der Marel, R. P., \& Vacca, W. D. 1999, AJ, 118, 831

Bransford, M. A., Appleton, P. N, Heisler, C. A., Norris, R. P., \& Marston, A. P. 1998, ApJ, 497, 133

Buta, R., \& Combes, F. 1996, Fundam. Cosmic Phys., 17, 95

Buta, R., Crocker, D. A., \& Byrd, G. G. 1999, AJ, 118, 2071

Buta, R., Treuthardt, P., Byrd, G. G., \& Crocker, D. A. 2000, AJ, 120, 1289

Carral, P., Turner, J. L., \& Ho, P. T. P. 1990, ApJ, 362, 434

Clark, B. G. 1980, A\&A, 89, 377

Colbert, E. J. M., Wilson, A. S., \& Bland-Hawthorn, J. 1994, ApJ, 436, 89

Colina, L., Alberdi, A., Torrelles, J. M., Panagia, N., \& Wilson, A. S. 2001, ApJ, 553, L19

Collison, P. M., Saikia, D. J., Pedlar, A., Axon, D. J., \& Unger, S. W. 1994, MNRAS, 268, 203
Condon, J. J. 1980, ApJ, 242, 894

Condon, J. J., \& Broderick, J. J. 1988, AJ, 96, 30

Condon, J. J., Condon, M. A., Gisler, G., \& Puschell, J. J. 1982, ApJ, 252, 102

Condon, J. J., Frayer, D. T., \& Broderick, J. J. 1991, AJ, 101, 362

Condon, J. J., Helou, G., Sanders, D. B., \& Soifer, B. T. 1990, ApJS, 73, 359

Conselice, C. J. 2003, ApJS, 147, 1

Contopoulos, C. 1975, ApJ, 201, 566

Crosthwaite, L. P., Turner, J. L., \& Ho, P. T. P. 2000, AJ, 119, 1720

Crosthwaite, L. P., Turner, J. L., Hurt, R. L., Levine, D. A., Martin, R. N., \& Ho, P. T. P. 2001, AJ, 122, 797

Davies, R. I., Sugai, H., \& Ward, M. J. 1997, MNRAS, 291, 314

de Vaucouleurs, G., de Vaucouleurs, A., Corwin, H. G., Jr., Buta, R. J., Paturel, G., \& Fouque, P. 1991, Third Reference Catalogue of Bright Galaxies (New York: Springer)

Eckart, A., Cameron, M., Boller, T., Krabbe, A., Blietz, M., Nakai, N., Wagner, S. J., \& Sternberg, A. 1996, ApJ, 472, 588

Elmegreen, D. M., Chromey, F. R., Santos, M., \& Marshall, D. 1997, AJ, 114, 1850

Elmegreen, D. M., Chromey, F. R., Sawyer, J. E., \& Reinfeld, E. L. 1999, AJ, 118,777

Elmegreen, D. M., Chromey, F. R., \& Warren, A. R. 1998, AJ, 116, 2834

Eracleous, M., Livio, M., \& Binette, L. 1995, ApJ, 445, L1

Faber, S. M., \& Gallagher, J. S. 1976, ApJ, 204, 365

Forbes, D. A., \& Norris, R. P. 1998, MNRAS, 300, 757

Fragile, P. C., Murray, S. D., Anninos, P., \& van Breugel, W. 2004, ApJ, 604, 74

Gallimore, J. F., \& Beswick, R. 2004, AJ, 127, 239

Genzel, R., Weitzel, L., Tacconi-Garman, L. E., Blietz, M., Cameron, M., Krabbe, A., \& Lutz, D. 1995, ApJ, 444, 129

Gerssen, J., van der Marel, R. P., Axon, D., Mihos, J. C., Hernquist, L., \& Barnes, J. E. 2004, AJ, 127, 75

Gonçalves, A. C., Véron-Cetty, M.-P., \& Véron, P. 1999, A\&AS, 135, 437

Grosbøl, P. 1985, A\&AS, 60, 261

Heller, C. H., \& Shlosman, I. 1996, ApJ, 471, 143

Ho, L. C., Filippenko, A. V., \& Sargent, W. L. W. 1997a, ApJ, 487, 568 1997b, ApJS, 112, 315

Ho, L. C., \& Ulvestad, J. S. 2001, ApJS, 133, 77

Högbom, J. A. 1974, A\&AS, 15, 417

Hummel, E., van der Hulst, J. M., Keel, W. C., \& Kennicutt, R. C., Jr. 1987, A\&AS, 70, 517

Jogee, S., Scoville, N. Z., \& Kenney, J. D. P. 2005, ApJ, 630, 837

Keel, W. C. 1983, ApJS, 52, 229 
Knapen, J. H. 2005, A\&A, 429, 141

Knapen, J. H., Beckman, J. E., Heller, C. H., Shlosman, I., \& de Jong, R. S. 1995, ApJ, 454, 623

Komossa, S., Burwitz, V., Hasinger, G., Predehl, P., Kaastra, J. S., \& Ikebe, Y. 2003, ApJ, 582, L15

Kotilainen, J. K., Forbes, D. A., Moorwood, A. F. M., van der Werf, P. P., \& Ward, M. J. 1996a, A\&A, 313, 771

Kotilainen, J. K., Moorwood, A. F. M., Ward, M. J., \& Forbes, D. A. 1996b, A\&A, 305, 107

Kotilainen, J. K., Reunanen, J., Laine, S., \& Ryder, S. D. 2000, A\&A, 353, 834 2001, A\&A, 366, 439

Laine, S., \& Gottesman, S. T. 1998, MNRAS, 297, 1041

Laine, S., \& Heller, C. H. 1999, MNRAS, 308, 557

Laine, S., Kenney, J. D. P., Yun, M. S., \& Gottesman, S. T. 1999, ApJ, 511, 709

Laine, S., Shlosman, I., Knapen, J. H., \& Peletier, R. F. 2002, ApJ, 567, 97

Laine, S., van der Marel, R. P., Rossa, J., Hibbard, J. E., Mihos, J. C., Böker, T., \& Zabludoff, A. I. 2003, AJ, 126, 2717

Lal, D. V., Shastri, P., \& Gabuzda, D. C. 2004, A\&A, 425, 99

Lira, P., Ward, M. J., Zezas, A., \& Murray, S. S. 2002, MNRAS, 333, 709

Lonsdale, C. J., Lonsdale, C. J., Smith, H. E., \& Diamond, P. J. 2003, ApJ, 592, 804

Malkan, M. A., Gorjian, V., \& Tam, R. 1998, ApJS, 117, 25

Maoz, D., Barth, A. J., Ho, L. C., Sternberg, A., \& Filippenko, A. V. 2001, AJ, 121, 3048

Márquez, I., et al. 2004, A\&A, 416, 475

Martin, P., \& Friedli, D. 1997, A\&A, 326, 449

Martini, P., Regan, M. W., Mulchaey, J. S., \& Pogge, R. W. 2003, ApJ, 589, 774

Max, C. E., Canalizo, G., MacIntosh, B. A., Raschke, L., Whysong, D., Antonucci, R., \& Schneider, G. 2005, ApJ, 621, 738

Miles, J. W., Houck, J. R., Hayward, T. L., \& Ashby, M. L. N. 1996, ApJ, 465, 191

Mirabel, I. F., \& Sanders, D. B. 1988, ApJ, 335, 104

Morganti, R., Tsvetanov, Z. I., Gallimore, J., \& Allen, M. G. 1999, A\&AS, 137, 457

Nagar, N. M., Falcke, H., Wilson, A. S., \& Ho, L. C. 2000, ApJ, 542, 186

Neff, S. G., \& Hutchings, J. B. 1992, AJ, 103, 1746

Noguchi, M. 1988, A\&A, 203, 259

Nordgren, T. E., Chengalur, J. N., Salpeter, E. E., \& Terzian, Y. 1997, AJ, 114, 77

Pannuti, T. G. 2000, Ph.D. thesis, Univ. New Mexico

Pasquali, A., Gallagher, J. S., \& de Grijs, R. 2004, A\&A, 415, 103

Perez-Olea, D. E., \& Colina, L. 1996, ApJ, 468, 191

Pérez-Ramírez, D. M., Peletier, R. F., Knapen, J. H., Laine, S., Doyon, R., \& Nadeau, D. 2000, MNRAS, 317, 234

Pogge, R. 1989, ApJ, 345, 730

Rafanelli, P., Schulz, H., Barbieri, C., Komossa, S., Mebold, U., Baruffolo, A., \& Radovich, M. 1997, A\&A, 327, 901

Regan, M. W., Sheth, K., \& Vogel, S. N. 1999, ApJ, 526, 97

Reunanen, J., Kotilainen, J. K., Laine, S., \& Ryder, S. D. 2000, ApJ, 529, 853

Rubin, V. C., Thonnard, R., \& Ford, W. K. 1975, ApJ, 199, 31

Ryder, S. D., Knapen, J. H., \& Takamiya, M. 2001, MNRAS, 323, 663
Saikia, D. J., Pedlar, A., Unger, S. W., \& Axon, D. J. 1994, MNRAS, 270, 46 Sakamoto, K., Okumura, S. K., Ishizuki, S., \& Scoville, N. Z. 1999, ApJS, 124, 403

Sakamoto, K., Okumura, S., Minezaki, T., Kobayashi, Y., \& Wada, K. 1995, AJ, 110, 2075

Sanders, D. B., Scoville, N. Z., Sargent, A. I., \& Soifer, B. T. 1988, ApJ, 324, L55

Schinnerer, E., Eckart, A., \& Boller, T. 2000, ApJ, 545, 205

Schmitt, H. R., Kinney, A. L., Storchi-Bergmann, T., \& Antonucci, R. 1997, ApJ, 477, 623

Schmitt, H. R., Ulvestad, J. S., Antonucci, R. R. J., \& Kinney, A. L. 2001, ApJS, 132, 199

Sellwood, J. A., \& Wilkinson A., 1993, Rep. Prog. Phys., 56, 173

Shlosman, I., Begelman, M. C., \& Frank, J. 1990, Nature, 345, 679

Simkin, S. M., Su, H. J., \& Schwarz, M. P. 1980, ApJ, 237, 404

Smith, B. J., Struck, C., \& Nowak, M. A. 2005, AJ, 129, 1350

Smith, D. A., Herter, T., Haynes, M. P., \& Neff, S. G. 1999, ApJ, 510, 669

Smith, H. E., Lonsdale, C. J., \& Lonsdale, C. J. 1998, ApJ, 492, 137

Sramek, R. 1975, AJ, 80, 771

Stine, P. C. 1992, ApJS, 81, 49

Storchi-Bergmann, T., González Delgado, R. M., Schmitt, H. R., Cid Fernandes, R., \& Heckman, T. 2001, ApJ, 559, 147

Tacconi, L. J., Genzel, R., Tecza, M., Gallimore, J. F., Downes, D., \& Scoville, N. Z. 1999, ApJ, 524, 732

Tecza, M., Genzel, R., Tacconi, L. J., Anders, S., Tacconi-Garman, L. E., \& Thatte, N. 2000, ApJ, 537, 178

Thean, A. H. C., Gillibrand, T. I., Pedlar, A., \& Kukula, M. J. 2001, MNRAS, 327, 369

Thean, A., Pedlar, A., Kukula, M. J., Baum, S. A., \& O’Dea, C. P. 2000, MNRAS, 314, 573

Toomre, A. 1977, in The Evolution of Galaxies and Stellar Populations, ed. B. M. Tinsley \& R. B. Larson (New Haven: Yale Univ.), 401

Tully, R. B. 1988, Nearby Galaxies Catalog (Cambridge: Cambridge Univ. Press)

Turner, J. L., \& Ho, P. T. P. 1983, ApJ, 268, L79

1994, ApJ, 421, 122

Turner, J. L., \& Hurt, R. L. 1992, ApJ, 384, 72

Ulvestad, J. S., Wilson, A. S., \& Sramek, R. A. 1981, ApJ, 247, 419

Unger, S. W., Lawrence, A., Wilson, A. S., Elvis, M., \& Wright, A. E. 1987, MNRAS, 228, 521

van Albada, G. D., \& van der Hulst, J. M. 1982, A\&A, 115, 263

van der Werf, P. P., Genzel, R., Krabbe, A., Blietz, M., Lutz, D., Drapatz, S., Ward, M. J., \& Forbes, D. A. 1993, ApJ, 405, 522

Veilleux, S., Kim, D.-C., Sanders, D. B., Mazzarella, J. M., \& Soifer, B. T. 1995, ApJS, 98, 171

Vila, M. B., Pedlar, A., Davies, R. D., Hummel, E., \& Axon, D. J. 1990, MNRAS, 242, 379

Weedman, D. W., Feldman, F. R., Balzano, V. A., Ramsey, L. W., Sramek, R. A., \& Wuu, C.-C. 1981, ApJ, 248, 105

Wilson, A. S., Helfer, T. T., Haniff, C. A., \& Ward, M. J. 1991, ApJ, 381, 79

Yun, M. S., \& Hibbard, J. E. 2001, ApJ, 550, 104 